\title{
Liouville theorems and blow up behaviour in semilinear reaction diffusion systems
}

\author{
by
}

\section{ANDREUCCI 1,2}

Dipartimento di Metodi e Modelli Matematici per le Scienze Applicate, Universita La Sapienza, via A. Scarpa 16, 00181 Roma, Italy.

\section{A. HERRERO ${ }^{1,3}$ and J. J. L. VELÁzQUEZ ${ }^{1,3}$}

Departamento de Matemática Aplicada,

Facultad de Matemáticas, Universidad Complutense, 28040 Madrid Spain.

ABSTRACT. - This paper is concerned with positive solutions of the semilinear system:

$$
\begin{cases}u_{t}=\Delta u+v^{p}, & p \geq 1 \\ v_{t}=\Delta v+u^{q}, & q \geq 1\end{cases}
$$

which blow up at $x=0$ and $t=T<\infty$. We shall obtain here conditions on $p, q$ and the space dimension $N$ which yield the following bounds on the blow up rates:

$$
u(x, t) \leq C(T-t)^{-\frac{p+1}{p q-1}}, \quad v(x, t) \leq C(T-t)^{-\frac{q+1}{p q-1}},
$$

for some constant $C>0$. We then use (1) to derive a complete classification of blow up patterns. This last result is achieved by means of a parabolic Liouville theorem which we retain to be of some independent interest.

${ }^{1}$ Partially supported by NATO Grant CRG920126.

2 Partially supported by MURST $40 \%$ "Problemi non lineari ....".

${ }^{3}$ Partially supported by DGICYT Grant PB93-0438.

AMS (MOS) Subject Classification: 35 B 40, 35 B 45, 35 K 55, 35 K 57

Annales de l'Institut Henri Poincaré - Analyse non linéaire - 0294-1449 
Finally, we prove the existence of solutions of (S) exhibiting a type of asymptotics near blow up which is qualitatively different from those that hold for the scalar case.

Key words and phrases: Semilinear systems, Reaction diffusion equations, Asymptotic behaviour, Liouville theorems, A priori estimates.

\section{INTRODUCTION}

In recent years a considerable effort has been devoted to unraveling the structure of the blow up mechanism for equations of the type

$$
u_{t}=\Delta u+f(u) ; \quad f(u)=u^{p},(p>1), f(u)=e^{u}, \ldots
$$

(cf. for instance [28] for a recent survey). While some interesting questions concerning the asymptotics of solutions near blow up remain open as yet, a rather comprehensive picture of the possible blow up patterns is now available. Let us examine for instance the case where $f(u)=u^{p}$ with $p>1$ in the equation above, i.e., consider the scalar equation

$$
u_{t}=\Delta u+u^{p}, \quad p>1
$$

and assume that $u(x, t)$ is a positive solution of (1.1) in a strip $S_{T}=\mathbf{R}^{N} \times(0, T)$. Suppose further that $u(x, t)$ blows up at $x=0$, $t=T$, by which we mean that there exist sequences $\left\{x_{n}\right\},\left\{t_{n}\right\}$ such that $\lim _{n \rightarrow \infty} x_{n}=0, \lim _{n \rightarrow \infty} t_{n}=T$, and $\lim _{n \rightarrow \infty} u\left(x_{n}, t_{n}\right)=\infty$. A quick glance at equation (1.1) suggests that blow up should be driven by the reaction term $f(u)=u^{p}$, which in turn hints at a blow up rate of the type

$$
u(x, t) \leq C(T-t)^{-\frac{1}{p-1}}, \quad \text { for some } C>0 \text { and any } t<T \text {. }
$$

From now on, we shall restrict our attention to positive solutions of the Cauchy problem corresponding to (1.1) with, say, bounded initial values $u(x, 0)=u_{0}(x)$. Suppose also that $p$ is subcritical, i.e.,

$$
1<p<\frac{N+2}{N-2} \quad \text { if } N \geq 3 \quad \text { (any } p>1 \text { if } N=1,2 \text { ). }
$$

Then a complete classification of blow up patterns for (1.1) is known. In order to state the corresponding result, we need to introduce some 
notation. For $x=\left(x_{1}, \ldots, x_{N}\right)$ and $\alpha=\left(\alpha_{1}, \ldots, \alpha_{N}\right)$, we shall write $x^{\alpha}=$ $x_{1}^{\alpha_{1}} \cdots x_{N}^{\alpha_{N}}$. Set $H_{j}=c_{j} \tilde{H}_{j}\left(x_{j} / 2\right)$, where $c_{j}=\left(2^{j / 2}(4 \pi)^{1 / 4}(j !)^{1 / 2}\right)^{-1}$ and $\tilde{H}_{j}(s)$ is the standard $j^{\text {th }}$-Hermite polynomial; actually $c_{j}$ is a normalisation constant whose role will be made clear later on. Finally, for $x$ and $\alpha$ as before we shall write $H_{\alpha}(x)=H_{\alpha_{1}}\left(x_{1}\right) \cdots H_{\alpha_{N}}\left(x_{N}\right)$. Let us introduce now self-similar variables given by

$$
\begin{gathered}
u(x, t)=(T-t)^{-\frac{1}{p-1}} \Phi(y, \tau), \quad y=x(T-t)^{-\frac{1}{2}} \\
\tau=-\log (T-t) .
\end{gathered}
$$

Notice that the choice $\Phi(y, \tau)=(p-1)^{-\frac{1}{p-1}}$ corresponds to an explicit, homogeneous solution of (1.1). Then there holds:

THEOREM A. - Let $u(x, t)$ be a positive solution of (1.1) in a strip $S_{T}=\mathbf{R}^{N} \times(0, T)$ which blows up at $x=0, t=T<\infty$, and assume that (1.2) and (1.3) hold. Let $\psi(y, \tau)$ be the function given by

$$
\Phi(y, \tau)=(p-1)^{-\frac{1}{p-1}}+\psi(y, \tau)
$$

where $\Phi$ is defined in (1.4). Then, if $\psi(\cdot, \tau) \not \equiv 0$ for some $\tau>0$, the following possibilitics arise. Either there exists an orthogonal transformation of coordinate axes such that, denoting still by $y$ the new coordinates,

$$
\psi(y, \tau)=-\frac{C_{p}}{\tau} \sum_{k=1}^{\ell} H_{2}\left(y_{k}\right)+o\left(\frac{1}{\tau}\right), \quad \text { as } \tau \rightarrow \infty
$$

where $1 \leq \ell \leq N$ and $C_{p}=(4 \pi)^{1 / 4}(p-1)^{-\frac{1}{p-1}}(\sqrt{2} p)^{-1}$, or there exists an even number $m, m \geq 4$, and constants $c_{\alpha} \equiv\left(c_{\alpha_{1}}, \ldots, c_{\alpha_{N}}\right)$ not all zero such that

$$
\psi(y, \tau)=-e^{\left(1-\frac{m}{2}\right) \tau} \sum_{|\alpha|=m} c_{\alpha} H_{\alpha}(y)+o\left(e^{\left(1-\frac{m}{2}\right) \tau}\right), \quad \text { as } \tau \rightarrow \infty
$$

where the homogeneous multilinear form $B(x)=\sum_{|\alpha|=m} c_{\alpha} x^{\alpha}$ is nonnegative. Convergence in (1.6), (1.7) takes place in $C_{\mathrm{loc}}^{k, \gamma}\left(\mathbf{R}^{N}\right)$ for any $k \geq 0$ and $\gamma \in(0,1)$.

See [26] and also [15]. Notice that different blow up patterns are known to exist; this was shown in [22] for the case $N=1$. Corresponding results for higher dimensional problems can be found in [9] and in [1].

We shall briefly sketch next the main ideas leading to Theorem $\mathrm{A}$, since they provide a basic background for the argument to be developed here. 
Following Giga and Kohn ([19]) we change variables as in (1.4) to obtain that $\Phi(y, \tau)$ satisfies

$$
\Phi_{\tau}=\Delta \Phi-\frac{1}{2} y \nabla \Phi+\Phi^{p}-\frac{\Phi}{p-1} .
$$

It is shown in [19] that, if (1.4) holds, then, as $\tau \rightarrow \infty, \Phi(y, \tau)$ should approach a stationary solution of (1.8) above. It is then shown that, if (1.3) is satisfied, the only global, nonnegative and bounded solutions of

$$
\Delta \Phi-\frac{1}{2} y \nabla \Phi+\Phi^{p}-\frac{\Phi}{p-1}=0
$$

are the constants $\Phi^{+}=(p-1)^{-\frac{1}{p-1}}$ and $\Phi_{-}=0$, a Liouville theorem of elliptic type. As a matter of fact, the case where $\Phi_{-} \equiv 0$ should be ruled out (cf. [17] for the case where $N=1$ and [20] for a general argument covering the range (1.3)). At this juncture, one has that

$$
\begin{aligned}
& \Phi(y, \tau) \rightarrow(p-1)^{-\frac{1}{p-1}} \text { as } \tau \rightarrow \infty, \\
& \text { uniformly on compact sets }|y| \leq R
\end{aligned}
$$

This is the crucial startpoint towards deriving Theorem A. In order to obtain it, though, further elaboration is required. To proceed, one first defines $\psi(y, \tau)$ through (1.5) and considers the equation satisfied by $\psi$, namely

$$
\psi_{\tau}=\Delta \psi-\frac{1}{2} y \nabla \psi+\psi+f(\psi) \equiv A \psi+f(\psi)
$$

where

$$
f(\psi)=\left((p-1)^{-\frac{1}{p-1}}+\psi\right)^{p}-(p-1)^{-\frac{p}{p-1}}-\frac{p \psi}{p-1}
$$

so that $f(\psi)=O\left(\psi^{2}\right)$ as $\psi \rightarrow 0$. The next step consists in deriving the form of the asymptotics of solutions of (1.10) as $\tau \rightarrow \infty$. To this end, one takes advantage of the fact that operator $A$ in (1.10) is self-adjoint in $L_{w}^{2}\left(\mathbf{R}^{N}\right)$, where

$$
L_{w}^{2}\left(\mathbf{R}^{N}\right)=\left\{\left.g \in L_{\mathbf{l o c}}^{2}\left(\mathbf{R}^{N}\right)\left|\int_{\mathbf{R}^{N}}\right| g(s)\right|^{2} e^{-|y|^{2} / 4} \mathrm{~d} y<\infty\right\},
$$

which is a Hilbert space when endowed with the norm

$$
\|g\|_{2, w}^{2} \equiv\|g\|^{2}=\int_{\mathbf{R}^{N}}|g(s)|^{2} e^{-|y|^{2} / 4} \mathrm{~d} y \equiv\langle g, g\rangle .
$$

On the other hand, the domain of $A, D(A)$ is given by $H_{w}^{2}\left(\mathbf{R}^{N}\right)$, where for any $k=1,2, \ldots, H_{w}^{k}\left(\mathbf{R}^{N}\right)$ is defined as the space of those functions 
in $H_{\text {loc }}^{k}\left(\mathbf{R}^{N}\right)$ such that they and their derivatives up to order $k$ belong to $L_{w}^{2}\left(\mathbf{R}^{N}\right)$. Moreover, the spectrum of $A$ consists of the eigenvalues

$$
1-\frac{m_{1}+\cdots+m_{N}}{2}, \quad \text { where } m_{1}, m_{2}, \ldots, m_{N}=0,1,2, \ldots .
$$

and the corresponding eigenfunctions are

$$
H_{m_{1} m_{2} \cdots m_{N}}(y) \equiv H_{m_{1}}\left(y_{1}\right) \cdots H_{m_{N}}\left(y_{N}\right)
$$

where the polynomials $H_{j}\left(y_{j}\right)$ have been defined before. This is the way in which Hermite polynomials enter the proof of Theorem A. Incidentally, the constants $c_{j}$ appearing in the definition of the $H_{j}$ are selected so that $\left\|H_{n}(y)\right\|=1$ for any $n=0,1,2, \ldots$

The basic motivation for the work under consideration was our desire to understand how the previous approach could be extended to systems of equations, where the mechanism of singularity formation is much less understood than in the scalar case. See however [5], [6], [10], [14], [16] for recent work on blow up for parabolic systems. More precisely, we shall consider here the simple semilinear model

$$
\begin{aligned}
& u_{t}=\Delta u+v^{p}, \quad p>0, \\
& v_{t}=\Delta v+u^{q}, \quad q>0 .
\end{aligned}
$$

It is known ( $c f[12]$ ) that any nontrivial positive solution of (13) which is defined for all $x \in \mathbf{R}^{N}$ must necessarily blow up in finite time if

$$
p q>1, \quad \text { and } \quad \frac{\gamma+1}{p q-1} \geq \frac{N}{2}, \quad \text { where } \gamma=\max (p, q) \text {. }
$$

When one considers boundary value problems for (13), blow up may occur whenever $p q>1$, provided that the data are large enough: see for instance [13]. In case where such phenomenon appears, both functions $u(x, t)$ and $v(x, t)$ must blow up at the same time.

Our purpose here is to obtain an analogue of Theorem A for (1.13). It will turn out that even in the case where $p=q$, important differences arise with respect to the scalar case. Moreover, in the course of analysing (1.13) some interesting new facts will emerge even for the single equation (1.1). We shall make these statements precise where appropriate. Drawing a parallel with the program fulfilled for the semilinear equation (1.1), our first step will consist in deriving an estimate for the blow up rate in our case. Assuming that $(u(x, t), v(x, t))$ blows up at $t=T<\infty$, a quick glance at 
the first order system obtained from (1.13) by dropping the laplacean terms therein, leads to guessing the following natural analogues of (1.2)

$$
\begin{gathered}
u(x, t) \leq C(T-t)^{-\frac{p+1}{p_{i}-1}}, \quad v(x, t) \leq C(T-t)^{-\frac{4+1}{p q-1}} \\
\text { for some } C>0 .
\end{gathered}
$$

It is to be noticed, however, that we cannot expect (1.14) to hold unless some restrictions are imposed on $p$ and $q$. For instance, even in the scalar case (1.2) is known to fail for (1.1), if $N \geq 11$ and $p$ is large enough, so that $(1.3)$ is not satisfied ( $c f[23])$. We shall prove here the following result

THEOREM 1. - Let $(u(x, t), v(x, t))$ be a solution of (1.13) defined in $S_{T}=\mathbf{R}^{N} \times(0, T)$, which blows up at time $t=T<\infty$. Then the bounds (1.14) hold true in $S_{T}$ provided that

$p q>1$, and at least one of the following two inequalities holds,

$$
q(p N-2)_{+}<N+2, \quad p(q N-2)_{+}<N+2,
$$

where, as usual, for any real number $s$ we set $s_{+}=\max (s, 0)$.

It is worth noticing here that the upper bounds (1.14) have been obtained by Caristi and Mitidieri [10], under assumptions on $p$ and $q$ different from (1.15). These authors consider radially symmetric solutions of (1.13) that blow up at $x=0$ and $t=T$, in such a way that $u_{t}(0, t) \geq 0$ and $v_{t}(0, t) \geq 0$ for all $t<T$. They proceed by means of ODE-related techniques, thus extending Weissler's approach for the scalar case (see [30]). We shall follow a different strategy here, and will resort instead to classical regularity methods for parabolic equations. Such a technique has been used in [2], [4] to discuss existence and non existence of solutions to Cauchy problems, for a class of equations and systems including (1.13), in terms of the regularity of the corresponding initial values. See also [3], and the book [11] for a general outline of these techniques. We should also mention the book [25], where somewhat similar ideas can be found, which however do not seem to suit our purposes here.

The proof of Theorem 1 is to be found in Section 2 below. Let us remark on pass that the $L^{1}$-estimate in Lemma 2.1 therein (which holds for any $p>0$ and $q>0$ with $p q>1$ ) seems to be of independent interest.

Once the upper bounds (1.14) arc available, the path is open to pursue the existing trail for scalar equations. To this end, we rescale variables as follows

$$
u(x, t)=(T-t)^{-\frac{p+1}{p q-1}} \Phi(y, \tau), \quad v(x, t)=(T-t)^{-\frac{q+1}{p q-1}} \Psi(y, \tau),
$$

where $y=x(T-t)^{-1 / 2}, \quad \tau=-\log (T-t)$. 
One is then led to the system

$$
\begin{aligned}
& \Phi_{\tau}=\Delta \Phi-\frac{1}{2} y \nabla \Phi+\Psi^{p}-\left(\frac{p+1}{p q-1}\right) \Phi \\
& \Psi_{\tau}=\Delta \Psi-\frac{1}{2} y \nabla \Psi+\Phi^{q}-\left(\frac{q+1}{p q-1}\right) \Psi
\end{aligned}
$$

Our next task consists in characterizing global, bounded and nonnegative solutions of (1.17) defined for all times. Clearly (1.17) possess constant solutions, namely

$$
\begin{gathered}
\Phi \equiv \Psi \equiv 0, \text { and the positive solution }(\Phi, \Psi) \equiv(\Gamma, \gamma) \text { of } \\
\gamma^{p}=\Gamma \frac{p+1}{p q-1}, \quad \Gamma^{q}=\gamma \frac{q+1}{p q-1}
\end{gathered}
$$

We shall prove the following parabolic Liouville theorem

THEOREM 2. - Assume that (1.15) holds. Then there exists a continuous and positive function $\varepsilon$ defined in the interval $(1,(N+2) /(N-2))$ if $N \geq 3$ (resp. in $(1, \infty)$ if $N=1,2$ ), such that, if

$$
\begin{gathered}
\left|p-p_{0}\right|+\left|q-p_{0}\right|<\varepsilon\left(p_{0}\right), \quad \text { for some } p_{0} \text { satisfying } \\
1<p_{0}<\frac{N+2}{N-2}, \quad \text { if } N \geq 3 \quad \text { (any } p_{0}>1 \text { for } N=1,2 \text { ), }
\end{gathered}
$$

then any nonnegative and bounded solution of (1.17) which is defined for all $y \in \mathbf{R}^{N}$ and all $\tau \in \mathbf{R}$ is either one of the constant solutions of (1.18) or satisfies otherwise

$$
\begin{array}{r}
\|\Phi(\cdot, \tau)-\Gamma\|+\|\Psi(\cdot, \tau)-\gamma\| \rightarrow 0, \quad \text { as } \tau \rightarrow-\infty \\
\|\Phi(\cdot, \tau)\|+\|\Psi(\cdot, \tau)\| \rightarrow 0, \quad \text { as } \tau \rightarrow \infty
\end{array}
$$

where $(\Gamma, \gamma)$ is the positive constant solution in (1.18), and \|\| denotes the $L_{w}^{2}\left(\mathbf{R}^{N}\right)$-norm defined in $(1.11 b)$.

Notice the rather tight assumption (1.19) in the statement of Theorem 2. At this stage, we have been unable to significantly weaken such hypothesis.

As a last goal in this paper, we set out to classify the possible patterns which may develop near a blow up point. To this end, we first prove that. if $(u(x, t), v(x, t))$ blows up at $x=0, t=T<\infty$, then one must have

$$
\Phi(y, \tau) \rightarrow \Gamma, \quad \Psi(y, \tau) \rightarrow \gamma, \quad \text { as } \tau \rightarrow \infty .
$$

uniformly on compact sets $|y| \leq R<\infty$, where

$\Gamma$. yare the positive constant described in (1.18). 
We shall prove (1.21) in Section 4 (cf. Lemma 4.1 there). A natural step consists then in linearizing around $(\Gamma, \gamma)$ by setting

$$
\Phi(y, \tau)=\Gamma+\varphi(y, \tau), \quad \Psi(y, \tau)=\gamma+\psi(y, \tau)
$$

A quick computation reveals now that $(\varphi, \psi)$ satisfies

$$
\begin{aligned}
\varphi_{\tau} & =\Delta \varphi-\frac{1}{2} y \nabla \varphi-\left(\frac{p+1}{p q-1}\right) \varphi+p \gamma^{p-1} \psi+f_{1}(\psi) \\
& \equiv A_{1} \varphi+p \gamma^{p-1} \psi+f_{1}(\psi) \\
\psi_{\tau} & =\Delta \psi-\frac{1}{2} y \nabla \psi-\left(\frac{q+1}{p q-1}\right) \psi+q \Gamma^{q-1} \varphi+f_{2}(\varphi) \\
& \equiv A_{2} \psi+q \Gamma^{q-1} \varphi+f_{2}(\varphi)
\end{aligned}
$$

where $f_{i}(s)=O\left(s^{2}\right)$ as $s \rightarrow 0$ for $i=1,2$. We may write (1.23) in a more compact form as follows

$$
\left(\begin{array}{c}
\varphi_{\tau} \\
\psi_{\tau}
\end{array}\right)=\left(\begin{array}{cc}
A_{1} & p \gamma^{p-1} I \\
q \Gamma^{q-1} I & A_{2}
\end{array}\right)\left(\begin{array}{l}
\varphi \\
\psi
\end{array}\right)+\left(\begin{array}{l}
f_{1}(\psi) \\
f_{2}(\varphi)
\end{array}\right),
$$

where $I$ denotes the identity operator. Notice that the operator

$$
A-\left(\begin{array}{cc}
A_{1} & p \gamma^{p-1} I \\
q \Gamma^{q-1} I & A_{2}
\end{array}\right)
$$

is not self-adjoint in the natural functional frame $V=L_{w}^{2}\left(\mathbf{R}^{N}\right) \times L_{w}^{2}\left(\mathbf{R}^{N}\right)$, unless $p=q$. At any rate, we will be able to expand any element $\left(\begin{array}{c}f \\ g\end{array}\right) \in V$ in the form

$$
\left(\begin{array}{l}
f \\
g
\end{array}\right)=\sum_{\alpha} a_{\alpha}^{+} H_{\alpha} u_{+}+\sum_{\alpha} a_{\alpha}^{-} H_{\alpha} u_{-}
$$

where $u_{+}, u_{-}$is a basis in $\mathbf{R}^{2}$ consisting on unit vectors parallel respectively to

$$
\left(\begin{array}{c}
1 \\
\frac{q+1}{p q-1} \gamma^{1-p}
\end{array}\right), \quad\left(\begin{array}{c}
1 \\
-\frac{q(p+1)}{p q-1}\left(p \gamma^{p-1}\right)^{-1}
\end{array}\right) .
$$

Set now

$$
v(y, \tau)=\left(\begin{array}{l}
\varphi(y, \tau) \\
\psi(y, \tau)
\end{array}\right)
$$

We then prove 
THEOREM 3. - Assume that

The upper bounds (1.14) are satisfied,

and the conclusion in Theorem 2 holds, i.e.,

Any nonnegative and bounded solution of (1.17) which is defined for all $y \in \mathbf{R}^{N}$ and $\tau \in \mathbf{R}$ is given by the constant functions defined in (1.18) or satisfies otherwise (1.20)

Then, if $(\Phi, \Psi) \not \equiv(0,0)$, the following possibilities arise. There exists an orthogonal transformation of coordinate axes such that, denoting still by $y$ the new coordinates, one has that either

$$
v(\cdot, \tau)=-\frac{C}{\tau} u_{+} \sum_{k=1}^{\ell} H_{2}\left(y_{k}\right)+o\left(\frac{1}{\tau}\right), \quad \text { as } \tau \rightarrow \infty,
$$

where $1 \leq \ell \leq N$ and $C=C(p, q)>0$, or else there exists an even number $m$, and constants $c_{\alpha}$ not all zero such that

$$
v(\cdot, \tau)=-\left(\sum_{|\alpha|=m} c_{\alpha} H_{\alpha}(y)\right) e^{\left(1-\frac{m}{2}\right) \tau} u_{+}+o\left(e^{\left(1-\frac{m}{2}\right) \tau}\right), \quad \text { as } \tau \rightarrow \infty
$$

where $m=4,6, \ldots$, or

$$
v(\cdot, \tau)=\left(\sum_{|\alpha|=m} c_{\alpha} H_{\alpha}(y)\right) e^{-\sigma \tau} u_{-}+o\left(e^{-\sigma \tau}\right), \quad \text { as } \tau \rightarrow \infty
$$

where $\sigma=\frac{(p+1)(q+1)}{p q-1}+\frac{m}{2}, m=0,1,2, \ldots$, the multilinear form $B(x)=\sum_{|\alpha|=m} c_{\alpha} x^{\alpha}$ is nonnegative in (1.28), and convergence in (1.27)(1.29) takes place in $H_{w}^{1}\left(\mathbf{R}^{N}\right)$ as well as in $C_{\mathrm{loc}}^{k, \gamma}\left(\mathbf{R}^{N}\right)$ for any $k \geq 0$ and $\gamma \in(0,1)$.

If we compare Theorem $\mathrm{A}$ and Theorem 3 , we readily observe that the main novelty in this last result consists in the possible occurrence of behaviours (1.29). We shall prove that such profiles actually exist. More precisely, we show here the following

THEOREM 4. - For any even number $m \geq 2$ and any dimension $N \geq 1$ there exist radial solutions of the Cauchy problem corresponding to (1.13) with arbitrary $p>1$ and $q>1$, such that they blow up at a given $T<\infty$, and (1.29) holds.

It follows from Theorem 4 that, even when $p=q$, there always are blow up profiles for (1.13) which are different from those occurring in the scalar Vol. $14, \mathrm{n}^{\circ} 1-1997$. 
case. For instance, in variables $(\Phi, \Psi)$ one has in a region $|y|=O(1)$ the following approximate picture for $m=8$ and $\Gamma<\gamma$

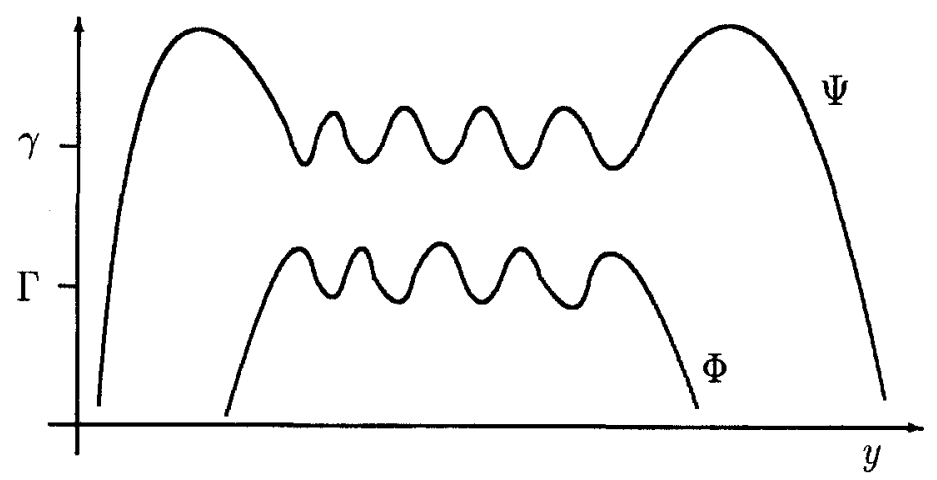

FIG 1. - Blow up patterns for case $(1.29)$.

Finally, the plan of the paper is as follows. A number of a priori estimates on solutions of (1.13) that will eventually lead to the proof of Theorem 1 are collected in the next Section 2. The following Section 3 contains a parabolic Liouville theorem, as well as the detail of our basic functional frame. These tools are used therein to prove Theorem 2. Finally, Theorems 3 and 4 make the content of Section 4.

\section{A PRIORI ESTIMATES. THE PROOF OF THEOREM 1}

In this section we shall consider the system

$$
\begin{aligned}
& u_{t}=\Delta u+v^{p}, \\
& v_{t}=\Delta v+u^{q},
\end{aligned}
$$

where $p$ and $q$ satisfy

$$
p>0, \quad q>0, \quad \text { and } \quad p q>1
$$

More precisely, we shall consider local positive solutions of (2.1), (2.2). By this we mean functions $u(x, t), v(x, t)$ which satisfy $(2.1),(2.2)$ in cylinders $Q_{T}=\Omega \times(0, T), \Omega$ being a bounded, smooth and open set in $\mathbf{R}^{N}(N \geq 1)$, and are positive in $Q_{T}$. 


\section{1. $L^{1}$-estimates}

For any $R>0$ and any locally integrable function $f$ we shall writc

$$
f_{B_{R}} f \mathrm{~d} x=\frac{1}{\left|B_{R}\right|} \int_{B_{R}} f \mathrm{~d} x,
$$

where $B_{R}=\{x|| x \mid<R\}$, and $\left|B_{R}\right|$ denotes the volume of the ball $B_{R}$. We then have

LEMMA 2.1. - Let $(u(x, t), v(x, t))$ be a local positive solution of (2.1), (2.2) defined in a cylinder $Q_{T}=\Omega \times\left(0, I^{\prime}\right), ' T<\infty$. Then for any $\rho>0$ such that $\rho^{2} \leq T-t$ and $B_{2 \rho} \subset \Omega$, there holds

$$
\begin{aligned}
& f_{B_{\rho}} u(x, t) \mathrm{d} x \leq \gamma \rho^{-2 \frac{p+1}{p q-1}} \\
& f_{B_{\rho}} v(x, t) \mathrm{d} x \leq \gamma \rho^{-2 \frac{q+1}{p q-1}}
\end{aligned}
$$

for some $\gamma=\gamma(N, p, q)>0$.

We shall point out a few consequences of Lemma 2.1. First, we observe that it implies in particular

Corollary 2.2. - Set $\rho^{2}=C(T-t)$ for some $C>0$. Then

$$
\begin{aligned}
& f_{B_{n(t)}} u(x, t) \mathrm{d} x \leq \gamma(T-t)^{-\frac{p+1}{p q-1}}, \\
& f_{B_{\rho(t)}} v(x, t) \mathrm{d} x \leq \gamma(T-t)^{-\frac{q+1}{p q-1}},
\end{aligned}
$$

for some $\gamma=\gamma(C, N, p, q)>0$, provided that $B_{2 \rho} \subset \Omega$.

On the other hand, Lemma 2.1 has some interesting consequences for the scalar equation (1.1). Indeed, if one sets $p=q$ and considers (1.1) as a particular case of (2.1), we obtain

Corollary 2.3. - If $u(x, t)$ is a local positive solution of (1.1) in $Q_{T}$ and $p>1$, there holds

$$
f_{B_{\rho}} u(x, t) \mathrm{d} x \leq \gamma(T-t)^{-\frac{1}{p-1}},
$$

for some $\gamma>0$, where $\rho^{2}(t)=(T-t)$, provided that $B_{2 \rho(t)} \subset \Omega$.

It is worth noticing that, while (2.5) seems to suggest that (1.2) should hold for all $p>1$, this last result is actually false. More Vol. $14, n^{\circ} 1-1997$. 
precisely, it has been recently shown in [23] that if $N \geq 11$ and $p>\left(N-2(N-1)^{1 / 2}\right)\left((N-4)-2(N-1)^{1 / 2}\right)^{-1}$, there exist positive and radial solutions of (1.1) such that

$$
\limsup _{t \rightarrow T}(T \cdots t)^{p-1} u(0, t)=\infty .
$$

By Corollary 2.3, however, such solutions must satisfy (2.5).

Proof of Lemma 2.1. - For fixed $\rho>0$, let $w(x)$ be the first eigenfunction of the problem

$$
-\Delta w=\lambda w, \quad \text { in } B_{\rho} ; \quad w=0, \quad \text { on }|x|=\rho,
$$

which satisfies the condition $\int_{B_{\rho}} w(x) \mathrm{d} x=1$. We may assume that $w(x)>0$ in $B_{\rho}$. By classical results one has that the first eigenvalue $\lambda_{1}$ is such that $\lambda_{1}=\gamma \rho^{-2}$ for some $\gamma=\gamma(N)$. We also have that $w \geq \gamma_{o} \rho^{-N}$ for $|x| \leq \rho / 2$, and some $\gamma_{o}=\gamma_{o}(N)$. Let us write

$$
U(t)=\int_{B_{\rho}} u(x, t) w(x) \mathrm{d} x, \quad V(t)=\int_{B_{\rho}} v(x, t) w(x) \mathrm{d} x .
$$

Assume first that $p, q \geq 1$. Then, if we multiply both sides of $(2.1 a)$ (resp. (2.1b)) by $w(x)$, integrate over $B_{\rho}$ and use Jensen's inequality, we readily arrive at

$$
\begin{aligned}
U^{\prime}(t) & \geq-\frac{\gamma}{\rho^{2}} U(t)+V(t)^{p}, \\
V^{\prime}(t) & \geq-\frac{\gamma}{\rho^{2}} V(t)+U(t)^{q},
\end{aligned}
$$

for $0<t<T$. Define now $y(t), z(t)$ as follows

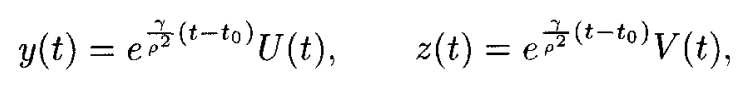

for a fixed $t_{0} \in(0, T)$. From now on, we shall denote by $\gamma$ a generic positive constant (possibly changing from line to line) depending at most on $N, p$ and $q$. Taking into account (2.7), one readily sees that

$$
y^{\prime} \geq e^{-\frac{\gamma}{\rho^{2}}\left(t-t_{0}\right)} z^{p}, \quad z^{\prime} \geq e^{-\frac{\gamma}{\rho^{2}}\left(t-t_{0}\right)} y^{q} .
$$

A comparison argument reveals then that

$$
y(t) \geq \lambda(t), \quad z(t) \geq \mu(t), \quad \text { for } t_{0}<t<T,
$$


where $\lambda, \mu$ are the solutions to the system

$$
\begin{gathered}
\lambda^{\prime}=e^{-\frac{\gamma}{\rho^{2}}\left(t-t_{0}\right)} \mu^{p}, \quad \mu^{\prime}=e^{-\frac{\gamma}{\rho^{2}}\left(t-t_{0}\right)} \lambda^{q}, \\
\lambda\left(l_{0}\right)=U_{0}, \mu\left(t_{0}\right)-V_{0}, \quad \text { where } U_{0}=U\left(t_{0}\right), V_{0}=V\left(t_{0}\right) .
\end{gathered}
$$

Notice that, on setting $\sigma(t)=\lambda(t)^{q+1}(q+1)^{-1}-\mu(t)^{p+1}(p+1)^{-1}$, we obtain that $\sigma^{\prime}(t) \equiv 0$, hence $\sigma(t)=\sigma\left(t_{0}\right)$ for any $t>t_{0}$. Thus

$$
\mu(t)=\left(\left(\frac{1+p}{1+q}\right)\left(\lambda(t)^{1+q}-U_{0}^{1+q}\right)+V_{0}^{1+p}\right)^{\frac{1}{1+p}} .
$$

We may substitute now (2.9) into the first equation in (2.8a) to obtain after one quadrature that

$$
\begin{aligned}
& \int_{U_{0}}^{\lambda(t)}\left(\left(\frac{1+p}{1+q}\right)\left(s^{1+q}-U_{0}^{1+q}\right)+V_{0}^{1+p}\right)^{-\frac{p}{1+p}} \mathrm{~d} s \\
& =\frac{\rho^{2}}{\gamma}\left(1-e^{-\frac{\gamma}{\rho^{2}\left(t-t_{0}\right)}}\right)
\end{aligned}
$$

which holds for any $t \in\left(t_{0}, T\right)$. We now impose that $\rho^{2} \leq T-t_{0}$, in which case we may select $t=t_{0}+\rho^{2}$ in (2.10) to obtain

$$
\begin{aligned}
\rho^{2} & \leq \gamma \int_{U_{0}}^{\lambda(t)}\left(\left(\frac{1+p}{1+q}\right)\left(s^{1+q}-U_{0}^{1+q}\right)+V_{0}^{1+p}\right)^{-\frac{p}{1+p}} \mathrm{~d} s \\
& \leq \gamma \int_{U_{0}}^{\infty}\left(s^{1+q}-U_{0}^{1+q}\right)^{-\frac{p}{1+p}} \mathrm{~d} s \leq \gamma U_{0}^{-\frac{p q-1}{p+1}} \int_{1}^{\infty}\left(r^{1+q}-1\right)^{-\frac{p}{1+p}} \mathrm{~d} r
\end{aligned}
$$

and since the last integral above converges, we deduce that

$$
U_{0} \leq \gamma \rho^{-2 \frac{p+1}{p q-1}} .
$$

The corresponding estimate for $V_{0}$ is obtained in the same way, and (2.3a) follows in this case.

Let us turn to the case where $\min (p, q)<1$. Without loss of generality, we may assume $q>1>p$ and $p q>1$. We shall exploit the following fact, established in [13], Lemma 4.1: There exists a positive increasing function $g \in C^{2}\left(\left(t_{0}, T\right)\right)$ such that

$$
\begin{aligned}
& g\left(t_{0}\right)=U_{0}, \\
& \left(\left(t-t_{0}\right)^{1-p} g^{\prime}(t)\right)^{\prime} \geq e^{-\frac{\gamma}{\rho^{2}}\left(t-t_{0}\right)} g(t)^{p q}, \quad \text { for } t_{0}<t<T .
\end{aligned}
$$


Note that, although the authors in [13] considered a problem in a bounded domain, their result is easily seen to carry over to our case by means of comparison arguments. Consider first the case where $5 \rho^{2}<T-t_{0}$ and $B_{k, p} \subset \Omega$, for some $k=k(N)>2$ to be chosen presently. Standard calculations show that $(2.11)$ implies

$$
\begin{aligned}
\left(\left(t-t_{0}\right)^{1-p} g^{\prime}(t)\right)^{2} & \geq 2 \int_{t_{0}+\rho^{2}}^{t} e^{\frac{1}{p^{2}}\left(\tau-t_{0}\right)} g(\tau)^{p q}\left(\tau-t_{0}\right)^{1-p} g^{\prime}(\tau) \mathrm{d} \tau \\
& \geq \gamma \rho^{2\left(1-p^{\prime}\right)}\left(g(t)^{p q+1}-g\left(t_{0}+\rho^{2}\right)^{p q+1}\right)
\end{aligned}
$$

for all $\rho^{2} \leq t-t_{0} \leq 2 \rho^{2}$. This inequality yields at once a lower bound for $g^{\prime}(t)$. On integrating such estimate over $\left(t_{0}+\rho^{2}, t_{0}+2 \rho^{2}\right)$ one finds

$$
\begin{aligned}
\rho^{p+1} & \leq \gamma \int_{g\left(t_{0}+\rho^{2}\right)}^{g\left(t_{1}+2 \rho^{2}\right)}\left(s^{p q+1}-g\left(t_{0}+\rho^{2}\right)^{p q+1}\right)^{-1 / 2} \mathrm{~d} s \\
& \leq \gamma g\left(t_{0}+\rho^{2}\right)^{-\frac{\mu_{i}-1}{2}} \int_{1}^{\infty}\left(r^{p q+1}-1\right)^{-1 / 2} \mathrm{~d} r<\infty
\end{aligned}
$$

Taking into account that $g^{\prime} \geq 0$ (so that $g\left(t_{0}\right) \leq g\left(t_{0}+\rho^{2}\right)$ ), the bound for $U_{0}$ follows. The function $v$ can be estimated as follows. Using classical representation formulae for solutions of the linear heat equation, one readily checks that

$$
\begin{gathered}
v(x, t) \geq \gamma_{0}(N) f_{B_{i}} v\left(y, t_{0}\right) \mathrm{d} y . \text { for } x \in B_{\rho}, \rho^{2} \leq t-t_{0} \leq 2 \rho^{2} . \\
u(x, t) \geq \gamma_{0}(N) \rho^{2}\left(_{B_{i}, \times\left(t_{0}+\rho^{2}, t_{0}+2 \rho^{2}\right)} v(x, t)\right)^{p} \\
\text { for } x \in B_{\rho}, t-t_{0}=3 \rho^{2} .
\end{gathered}
$$

Actually the requirement that $B_{k,} \subset \Omega$ for some $k=k(N)$ large enough is needed to derive (2.12). If we combine (2.12) and the bound for $U_{0}$ previously obtained, we have that

$$
\begin{aligned}
f_{B_{p}} v\left(y, t_{0}\right) \mathrm{d} y & \leq \gamma\left(\rho^{-2} f_{B_{i}} u\left(y, t_{0}+3 \rho^{2}\right) \mathrm{d} y\right)^{1 / p} \\
& \leq \gamma\left(\rho^{-2\left(1+\frac{p+1}{p \eta-1}\right)}\right)^{1 / p}=\gamma \rho^{-2 \frac{q+1}{p-1}}
\end{aligned}
$$

Indeed the previous part of the proof applies since we have that $t_{0}+3 \rho^{2}<T-2 \rho^{2}$ by assumption. To conclude, we merely notice that the extra restrictions imposed above on $\rho$ can be relaxed to the assumptions made in the statement of the Lemma by means of the following 
straightforward covering argument. Let $\rho^{2} \leq(T-t), B_{\rho} \subset B_{2 \rho} \subset \Omega$. Then the ball $B_{\rho}$ can be covered by $\gamma(N) k^{N}$ balls $B^{i}$ of radius $\rho_{i}=\rho / k$ ( $k$ as above), centered at points of $B_{\rho}$. Therefore, for each $i$, the ball with the same center as $B^{i}$ and with radius $k \rho_{i}=\rho$ is contained in $\Omega$. As we may assume that $k \geq \sqrt{5}$, it is clear that $5 \rho_{i}^{2} \leq(T-t)$, so that the extra assumptions made above are satisfied. The bounds in (2.3) then follow by applying the just proven estimates to every ball $B^{i}$. Note that the final constant $\gamma$ appearing in (2.3) differs from the one found under the extra assumptions only for a multiplicative factor depending on $N$.

Taking into account (2.4), we may multiply both sides of $(2.1 a)$ and (2.1b) by the function $w$ introduced in (2.6), and then integrate over $B_{,} \times\left(t-\rho^{2}: t\right)$ to obtain

COROLlARY 2.4. - Under the assumptions of Lemma 2.1, one has that if $\rho^{2}=C(T-t)$ for some $C>0$, there exists $\gamma=\gamma(N, C, p, q)>0$ such that

$$
\begin{aligned}
& \int_{t-\rho^{2}}^{t} \int_{B_{\rho}} u^{q} \mathrm{~d} x \mathrm{~d} \tau \leq \gamma(T-t)^{\frac{N}{2}-\frac{u+1}{p^{\prime \prime}+1}}, \\
& \int_{t-\rho^{2}}^{t} \int_{B_{t^{\prime}}} v^{p} \mathrm{~d} x \mathrm{~d} \tau \leq \gamma(T-t)^{\frac{\nu}{2}-\frac{p+1}{j^{\prime \prime}}} .
\end{aligned}
$$

We point out that the arguments in the proof of Lemma 2.1 do not require of any smallness restriction on $T-t$. We then may let $t \rightarrow 0$ in (2.3) to obtain

COROLLARY 2.5. - Under the assumptions of Lemma 2.1, $u(x, t)$ has an initial trace $\mu$ in $\Omega, \mu$ being a locally finite Borel measure in $\Omega$. Moreover for all $x \subset \Omega$,

$$
\begin{aligned}
& \mu\left(B_{\rho}(x)\right) \leq \gamma \rho^{N-2 \frac{p+1}{p q-1}} \\
& \text { for } 0<\rho<\min \left(T^{1 / 2}, \frac{1}{2} \operatorname{dist}(x, \partial \Omega)\right) .
\end{aligned}
$$

A similar statement holds for $v(x, t)$.

Remark. - a) If $2(p+1)<N(p q-1),(2.15)$ actually places a restriction on the local regularity of any admissible initial datum $u(x, 0)$. Such restrictions are well known for the case of the scalar equation (1.1) (see for instance [4], [3], and the references therein). Concerning the case of the system at hand, we refer to [2], where existence of solutions is proved under assumptions which, in the light of Corollary 2.5 above, are optimal if $2(p+1) \leq N(p q-1), 2(q+1) \leq N(p q-1)$. 
b) Assume that one of the two inequalities stated at the end of part a) above is violated. Then, by letting $\rho \rightarrow \infty$ in (2.15) we would recover that the only global solution of the system is the trivial one, i.e., $u \equiv v \equiv 0$, as observed in [12].

\section{2. $L^{\infty}$-estimates}

In this paragraph we shall derive some upper bounds for solutions of (2.1) which will eventually provide the proof of Theorem 1. To that end we begin by recalling some notation. For given positive constants $k, \rho, \sigma$ and $t$ with $\sigma<1$, and any nonnegative integer $n$, we set

$$
\begin{aligned}
& k_{n}=k\left(1-2^{-n-1}\right), \rho_{n}=\rho\left(1+\sigma 2^{-n}\right), t_{n}=\frac{t}{2}\left(1-\sigma 2^{-n}\right), \\
& B_{n}=B_{\rho_{n}}, Q_{n}=B_{n} \times\left(t_{n}, t\right), Q_{\infty}=B_{\rho} \times(t / 2, t),
\end{aligned}
$$

The key result in this Section is the following estimate for the linear equation which we deem to be of some independent interest. This estimate is valid for nonlinear equations too (see the Remark at the end of this Section). While the Lemma can be proved by the classical methods in [24], [11], we are unaware of any precise reference in the literature, and will therefore provide a proof below. The precise result reads as follows.

LEMMA 2.6. - Let $u(x, t)$ be a nonnegative solution of

$$
u_{t}=\Delta u+f, \quad \text { in } Q_{0},
$$

where $f \in L^{r}\left(Q_{0}\right)$ with $r>(N+2) / 2$. Then for any $\alpha>0$ there exists $\gamma=\gamma(N, r, \alpha)>0$ such that

$$
\begin{aligned}
\|u\|_{\infty_{2} Q_{\infty}} \leq & \gamma\left(1+\frac{t}{\rho^{2}}\right)^{\frac{N+2}{2}}\left(\frac{\rho^{2}}{t}\right)^{\frac{N}{2}} f \int_{Q_{0}} u \mathrm{~d} x \mathrm{~d} \tau \\
& +\gamma\|f\|_{r, Q_{0}}^{\nu_{1}}\left(\iint_{Q_{0}} u^{\alpha} \mathrm{d} x \mathrm{~d} \tau\right)^{\nu_{2}}
\end{aligned}
$$

where

$$
\nu_{1}=\frac{(N+2) r}{(N+2) r+\alpha(2 r-(N+2))}, \quad \nu_{2}=\frac{1-\nu_{1}}{\alpha} .
$$

Proof: - Let $\zeta_{n}$ be a smooth and nonnegative cut-off function which vanishes outside $Q_{n}$ and is such that $\zeta_{n} \equiv 1$ on $Q_{n+1},\left|\nabla \zeta_{n}\right| \leq\left(\gamma 2^{n}\right) /(\sigma \rho)$, and $0 \leq \zeta_{n_{t}} \leq\left(\gamma 2^{n}\right) /(\sigma t)$, where as before $\gamma$ will denote a positive generic constant, possibly changing from line to line, which depends at most on 
the parameters listed in the statement of the Lemma. We next use the customary notation $s_{+}=\max (s, 0)$, and for fixed $\beta>0$ multiply both sides of (2.17) by the test function $\left(u-k_{n+1}\right)_{+}^{\beta} \zeta_{n}{ }^{2}$. One then obtains after integration by parts that

$$
\begin{aligned}
& \sup _{t_{n}<\tau<t} \int_{B_{n}}\left(u-k_{n+1}\right)_{+}^{\beta+1}{\zeta_{n}}^{2} \mathrm{~d} x \\
& \quad+\int_{Q_{n}}\left|\nabla\left(\left(u-k_{n+1}\right)_{+}^{\frac{\beta+1}{2}} \zeta_{n}\right)\right|^{2} \mathrm{~d} x \mathrm{~d} \tau \\
& \leq \frac{2^{2 n} \gamma}{\sigma^{2}}\left(t^{-1}+\rho^{-2}\right) \int_{Q_{n}}\left(u-k_{n+1}\right)_{+}^{\beta+1} \mathrm{~d} x \mathrm{~d} \tau \\
& \quad+\gamma \int_{Q_{n}} f\left(u-k_{n+1}\right)_{+}^{\beta} \zeta_{n}^{2} \mathrm{~d} x \mathrm{~d} \tau
\end{aligned}
$$

Write now $A_{n+1}=\left\{(x, t) \in Q_{n} \mid u(x, t)>k_{n+1}\right\}$. Then for any $r>1$ one has that

$$
\begin{gathered}
\int_{Q_{n}}\left(u-k_{n+1}\right)_{+}^{\beta+1} \mathrm{~d} x \mathrm{~d} \tau \\
\leq\left|A_{n+1}\right|^{1 / r}\left(\int_{Q_{n}}\left(u-k_{n}\right)_{+}^{\frac{(\beta+1) r}{r-1}} \mathrm{~d} x \mathrm{~d} \tau\right)^{\frac{r-1}{r}}, \\
\int_{Q_{n}} f\left(u-k_{n+1}\right)_{+}^{\beta} \zeta_{n}{ }^{2} \mathrm{~d} x \mathrm{~d} \tau \leq \gamma 2^{n} k^{-1} \int_{Q_{n}}|f|\left(u-k_{n}\right)_{+}^{\beta+1} \mathrm{~d} x \mathrm{~d} \tau \\
\leq \gamma 2^{n} k^{-1}\left(\int_{Q_{n}}|f|^{r} \mathrm{~d} x \mathrm{~d} \tau\right)^{1 / r}\left(\int_{Q_{n}}\left(u-k_{n}\right)_{+}^{\frac{(\beta+1) r}{r-1}} \mathrm{~d} x \mathrm{~d} \tau\right)^{\frac{r-1}{r}} .
\end{gathered}
$$

Set now $s=(N+2)(r-1) /(N r)>1$. Then

$$
\begin{aligned}
& \iint_{Q_{n+1}}\left(u-k_{n+1}\right)_{+}^{\frac{(\beta+1) r}{r-1}} \mathrm{~d} x \mathrm{~d} \tau \\
& \quad \leq \int_{Q_{n}}\left(\left(u-k_{n+1}\right)_{+}^{\frac{\beta+1}{2}} \zeta_{n}\right)^{2 \frac{r}{r-1}} \mathrm{~d} x \mathrm{~d} \tau \\
& \quad \leq\left|A_{n+1}\right|^{\frac{\beta-1}{s}}\left(\int_{Q_{n}}\left(\left(u-k_{n+1}\right)_{+}^{\frac{\beta+1}{2}} \zeta_{n}\right)^{2 \frac{N+2}{N}} \mathrm{~d} x \mathrm{~d} \tau\right)^{1 / s} .
\end{aligned}
$$


We now take advantage of (2.19)-(2.21), and of the embedding in [24] p. 74 to obtain

$$
\begin{aligned}
& \iint_{Q_{n+1}}\left(u-k_{n+1}\right)_{+}^{\frac{(\beta+1), r}{r-1}} \mathrm{~d} x \mathrm{~d} \tau \\
& \leq \gamma\left|A_{n+1}\right|^{\frac{s-1}{\varepsilon}}\left(\int_{Q_{n}}\left(u-k_{n}\right)_{+}^{\frac{(\beta+1) r}{r-1}} \mathrm{~d} x \mathrm{~d} \tau\right) \\
& \quad \times\left(2^{2 n} \sigma^{-2}\left(t^{-1}+\rho^{-2}\right)\left|A_{n+1}\right|^{1 / r}+2^{n} k^{-1}\|f\|_{r, Q_{n}}\right)^{\frac{r}{r-1}}
\end{aligned}
$$

If we now write $m=(\beta+1) r /(r-1)$, and observe that for all $\alpha>0$

$$
\left|A_{n+1}\right| \leq \gamma k^{-\alpha} 2^{n \alpha} \int_{Q_{n}}\left(u-k_{n}\right)_{+}^{\alpha} \mathrm{d} x \mathrm{~d} \tau,
$$

then it readily follows from (2.22) that

$$
\begin{aligned}
& \iint_{Q_{n+1}}\left(u-k_{n+1}\right)_{+}^{\frac{(\beta+1) \cdot}{r-1}} \mathrm{~d} x \mathrm{~d} \tau \\
& \leq \gamma 2^{\gamma n} k^{-\delta_{1}}\left(\int_{Q_{n}}\left(u-k_{n}\right)_{+}^{\frac{(3+1) \gamma}{r-1}} \mathrm{~d} x \mathrm{~d} \tau\right)^{1+\frac{s-1}{s}} \\
& \times\left(k^{-\frac{m}{r-1}}\|u\|_{m, Q_{n}}^{\frac{3 n}{r-1}}\left(\sigma^{-2}\left(t^{-1}+\rho^{-2}\right)\right)^{\frac{r}{r-1}}+k^{-\frac{r}{r-1}}\|f\|_{r \cdot Q_{n}}^{\frac{r}{r-1}}\right),
\end{aligned}
$$

where $\delta_{1}=(\beta+1)(s-1) r /(s(r-1))$. A classical argument $(c f$ [24] p. 95) yields then that

$$
\|u\|_{\infty, Q_{\infty}} \leq k
$$

provided that

$$
\begin{aligned}
& \gamma \iint_{Q_{0}} u^{\frac{(\beta+1) r}{r+1}} \mathrm{~d} x \mathrm{~d} \tau \\
& \quad=k^{\frac{(\beta+1) r}{r-1}}\left(k^{-\frac{m}{p-1}}\|u\|_{m, Q_{0}}^{\frac{n n}{r-1}}\left(\sigma^{-2}\left(t^{-1}+\rho^{-2}\right)\right)^{\frac{r}{r-1}}+k^{-\frac{r}{r-1}}\|f\|_{r, Q_{0}}^{\frac{r}{r-1}}\right)^{-\frac{s}{s-1}} .
\end{aligned}
$$

whence

$$
\begin{aligned}
& \|u\|_{\infty, Q_{\infty}}^{\frac{(\beta+1) r}{r-1}} \leq \gamma \iint_{Q_{U}} u^{\frac{(\beta+1) r}{r+1}} \mathrm{~d} x \mathrm{~d} \tau\left(\left(\frac{\|u\|_{m, Q_{0}}}{\|u\|_{\infty, Q_{\infty}}}\right)^{\frac{r u}{r-1}}\left(\sigma^{-2}\left(t^{-1}+\rho^{-2}\right)\right)^{\frac{r}{r-1}}\right. \\
& \left.\quad+\left(\frac{\|f\|_{r, Q_{0}}}{\|u\|_{\infty, Q_{\infty}}}\right)^{\frac{r}{r-1}}\right)^{\frac{s}{s-1}} \equiv \gamma \iint_{Q_{0}} u^{\frac{(\beta+1) r}{r-1}} \mathrm{~d} x \mathrm{~d} \tau(A+B)
\end{aligned}
$$


Suppose first that $A \geq B$. Setting

$$
\delta_{2}=\frac{(\beta+1) r}{r-1}+\frac{m}{r-1} \frac{s}{s-1}=\frac{2(\beta+1) r^{2}}{(r-1)(2 r-(N+2))},
$$

one then has that

$$
\begin{aligned}
\|u\|_{\infty, Q_{\infty}}^{\delta_{2}} & \leq \gamma\left(\sigma^{-2}\left(t^{-1}+\rho^{-2}\right)\right)^{\frac{(N+2) r}{2 r}}\left(\iint_{Q_{0}} u^{\frac{(\beta+1) r}{r-1}} \mathrm{~d} x \mathrm{~d} \tau\right)^{\frac{2 r}{2 r-(N+2)}} \\
& \leq \gamma\left(\sigma^{-2}\left(t^{-1}+\rho^{-2}\right)\right)^{\frac{(N+2) r}{2 r-(N+2)}}\|u\|_{\infty, Q_{0}}^{\delta_{3}}\left(\iint_{Q_{0}} u \mathrm{~d} x \mathrm{~d} \tau\right)^{\frac{2 r}{2 r-(N+2)}} .
\end{aligned}
$$

where

$$
\delta_{3}=\left(\frac{(\beta+1) r}{r-1}-1\right) \frac{2 r}{2 r-(N+2)}=\frac{2 r(\beta r+1)}{(r-1)(2 r-(N+2))} .
$$

Hence

$$
\|u\|_{\infty, Q_{\infty}} \leq \gamma\|u\|_{\infty, Q_{0}}^{\frac{3 r+1}{\beta r+r}}\left(\sigma^{-2}\left(t^{-1}+\rho^{-2}\right)\right)^{\frac{(N+2)(r-1)}{2(\beta+1) \gamma}}\left(\iint_{Q_{0}} u \mathrm{~d} x \mathrm{~d} \tau\right)^{\frac{r-1}{(\beta+1) r}}
$$

and thus

$$
\|u\|_{\infty, Q_{\infty}} \leq \nu\|u\|_{\infty, Q_{0}}+\gamma \nu^{-\frac{\beta r+1}{r-1}}\left(\sigma^{-2}\left(t^{-1}+\rho^{-2}\right)\right)^{\frac{N+2}{2}}\left(\iint_{Q_{0}} u \mathrm{~d} x \mathrm{~d} \tau\right),
$$

for any $\nu \in(0,1)$. Assume now that $B>A$ in $(2.25)$. Then, setting

$$
\delta_{4}=\frac{(\beta+1) r}{r-1}+\frac{r s}{(r-1)(s-1)}=\frac{(\beta+1) r}{r-1}+\frac{(N+2) r}{2 r-(N+2)},
$$

it follows that

$$
\begin{aligned}
\|u\|_{\infty, Q_{\infty}}^{\delta_{4}} & \leq \gamma\|f\|_{r, Q_{0}}^{\frac{(N+2) r}{2 r-(N+2)}} \iint_{Q_{0}} u^{\frac{(3+1) r}{r-1}} \mathrm{~d} x \mathrm{~d} \tau \\
& \leq \gamma\|f\|_{r, Q_{0}}^{\frac{(N+2) r}{2 r-(N+2)}}\|u\|_{\infty, Q_{0}}^{\frac{(\beta+1) r}{r-1}-\alpha} \iint_{Q_{0}} u^{\alpha} \mathrm{d} x \mathrm{~d} \tau
\end{aligned}
$$

provided that $\beta=\beta(r, \alpha)>0$ has been chosen large enough. Whence

$$
\|u\|_{\infty, Q_{\infty}} \leq \nu\|u\|_{\infty, Q_{0}}+\gamma \nu^{-\theta}\|f\|_{r, Q_{0}}^{\nu_{1}}\left(\iint_{Q_{0}} u^{\alpha} \mathrm{d} x \mathrm{~d} \tau\right)^{\nu_{2}}
$$


for any $\nu \in(0,1)$, with $\theta=\theta(\beta, r, \alpha, N)>0$ and $\nu_{1}, \nu_{2}$ as in (2.18). Putting together (2.26) and (2.27), we obtain that for any $\nu \in(0,1)$ there exists $C=C(\nu)>0$ so that

$$
\begin{aligned}
\|u\|_{\infty, Q_{\infty}} \leq \nu\|u\|_{\infty, Q_{0}} & +\gamma C\left(\sigma^{-2}\left(t^{-1}+\rho^{-2}\right)\right)^{\frac{N+2}{2}}\left(\iint_{Q_{0}} u \mathrm{~d} x \mathrm{~d} \tau\right) \\
& +\gamma C\|f\|_{r, Q_{0}}^{\nu_{1}}\left(\iint_{Q_{0}} u^{\alpha} \mathrm{d} x \mathrm{~d} \tau\right)^{\nu_{2}}
\end{aligned}
$$

We next select a sequence of cylinders $\left\{Q^{i}\right\}$ in the following form

$$
Q^{i}=B^{i} \times\left(t^{i}, t\right), \quad t^{i}=\frac{t}{2}\left(1-\left(1-2^{-i}\right) \sigma\right) ; \quad i=0,1,2, \ldots
$$

where

$$
B^{i}=B_{\rho^{i}}, \quad \rho^{i}=\rho\left(1+\left(1-2^{-i}\right) \sigma\right), \quad B^{0}=B_{\infty},
$$

so that $Q^{\infty}=Q_{0}$. If we write now (2.28) with $Q_{\infty}$ (resp. $Q_{0}$ ) replaced by $Q^{i}$ (resp. $Q^{i+1}$ ), we finally obtain by induction on $i$ that

$$
\begin{aligned}
\|u\|_{\infty, Q_{\infty}} \leq & \nu^{i}\|u\|_{\infty, Q^{i}} \\
& +\gamma C \sum_{j=0}^{i}\left(2^{N+2} \nu\right)^{j}\left(\sigma^{-2}\left(t^{-1}+\rho^{-2}\right)\right)^{\frac{N+2}{2}}\left(\iint_{Q_{0}} u \mathrm{~d} x \mathrm{~d} \tau\right) \\
& +\gamma C \sum_{j=0}^{i} \nu^{j}\|f\|_{r, Q_{0}}^{\nu_{1}}\left(\iint_{Q_{0}} u^{\alpha} \mathrm{d} x \mathrm{~d} \tau\right)^{\nu_{2}}
\end{aligned}
$$

whence the result, on letting $i \rightarrow \infty$, for a suitable choice of $\nu$.

We now point out the following consequence of Lemma (2.6).

COROLlary 2.7. - Let $u(x, t)$ be a positive solution of the scalar equation (1.1) in $Q_{0}$. Then, if $p<(N+2) / N$, there exists a constant $\gamma=\gamma(N, \rho, \sigma)$ such that

$\|u\|_{\infty, Q_{\infty}} \leq \gamma\left(1+\frac{t}{\rho^{2}}\right)^{\frac{N+2}{2}}\left(\frac{\rho^{2}}{t}\right)^{\frac{N}{2}} \oiiint_{Q_{0}} u \mathrm{~d} x \mathrm{~d} \tau+\gamma\left(\iint_{Q_{0}} u^{p} \mathrm{~d} x \mathrm{~d} \tau\right)^{\mu}$

where $\mu=2(N+2-N p)^{-1}$.

Proof. - Let $r>(N+2) / 2$. Clearly one has that

$$
\left\|u^{p}\right\|_{r, Q_{0}} \leq\|u\|_{\infty, Q_{0}}^{p \frac{r-1}{r}}\left(\iint_{Q_{0}} u^{p} \mathrm{~d} x \mathrm{~d} \tau\right)^{1 / r}
$$


Therefore, if we use (2.18a) with $f=u^{p}$ and $\alpha=p$, and set

$$
H \equiv H(\rho, t, \gamma)=\gamma\left(1+\frac{t}{\rho^{2}}\right)^{\frac{N+2}{2}}\left(\frac{\rho^{2}}{t}\right)^{\frac{N}{2}}
$$

it follows that

$$
\begin{aligned}
\|u\|_{\infty, Q_{\infty}} & \leq H \iint_{Q_{0}} u \mathrm{~d} x \mathrm{~d} \tau+\gamma\|u\|_{\infty, Q_{0}}^{p \nu_{1} \frac{r-1}{r}}\left(\iint_{Q_{0}} u^{p} \mathrm{~d} x \mathrm{~d} \tau\right)^{\nu_{2}+\nu_{1} / r} \\
& \leq H \iint_{Q_{0}} u \mathrm{~d} x \mathrm{~d} \tau+\nu\|u\|_{\infty, Q_{0}}+C\left(\iint_{Q_{0}} u^{p} \mathrm{~d} x \mathrm{~d} \tau\right)^{\mu}
\end{aligned}
$$

where we have used Young's inequality to derive the last bound above, $\nu \in(0,1)$ is to be chosen, $C$ depends on $\gamma$, and $\mu$ is as in the statement of the Corollary. An iterative argument similar to the one at the end of the proof of Lemma 2.6 yields the result.

We are now in a position to prove

LEMMA 2.8. - Let $(u, v)$ be a positive solution of (2.1) in the cylinder $Q_{0}$. If at least one of the two inequalities

$$
p(q N-2)_{+}<N+2, \quad \text { or } \quad q(p N-2)_{+}<N+2
$$

holds, then we have

$$
\begin{aligned}
\|u\|_{\infty, Q_{\infty}} \leq H & \iint_{Q_{0}} u \mathrm{~d} x \mathrm{~d} \tau+\left(H \iint_{Q_{0}} v \mathrm{~d} x \mathrm{~d} \tau\right)^{p(r-1) \mu_{1}} \\
& \times\left(\iint_{Q_{0}} u^{q} \mathrm{~d} x \mathrm{~d} \tau\right)^{\mu_{2}}\left(\iint_{Q_{0}} v^{p} \mathrm{~d} x \mathrm{~d} \tau\right)^{\mu_{1}} \\
& +\gamma\left(\iint_{Q_{0}} u^{q} \mathrm{~d} x \mathrm{~d} \tau\right)^{\frac{x_{2}}{1-x_{1}}}\left(\iint_{Q_{0}} v^{p} \mathrm{~d} x \mathrm{~d} \tau\right)^{\frac{x_{3}}{1-x_{1}}},(2.31 a)
\end{aligned}
$$

provided $r, s>(N+2) / 2$ are chosen so that

$$
\chi_{1}=p q(r-1)(s-1) \sigma_{1} \mu_{1}<1 .
$$

Here $\gamma=\gamma(N, p, q, r, s, \sigma)$,

$$
\begin{aligned}
& \mu_{1}=(N+2)((N+2) r+q(2 r-N-2))^{-1}, \\
& \sigma_{1}=(N+2)((N+2) s+p(2 s-N-2))^{-1},
\end{aligned}
$$




$$
\begin{gathered}
\chi_{2}=p(r-1) \sigma_{1} \mu_{1}+\mu_{2} . \quad \chi_{3}=\mu_{1}\left((r-1)\left(1-s \sigma_{1}\right)+1\right), \\
\mu_{2}=\left(1-\mu_{1} r\right) q^{-1}
\end{gathered}
$$

and $H=H(\rho, t, \gamma)>0$ has been defined in (2.29). Of course vatisfies an analogous estimate.

Proof. - We shall keep to the notation used in the proof of our previous Corollary 2.7 , and use first $(2.18 a)$ in $(2.1 a)$ with $f=v^{p}$ and $\alpha=q$. This yields

$$
\begin{aligned}
\|u\|_{\infty} Q_{\infty} \leq & H \iint_{Q_{0}} u \mathrm{~d} x \mathrm{~d} \tau+\gamma\left\|v^{\mu^{\prime}}\right\|_{r \cdot Q_{0}}^{\mu_{1} r}\left(\iint_{Q_{n}} u^{q} \mathrm{~d} x \mathrm{~d} \tau\right)^{\mu_{2}} \\
\leq & H \iint_{Q_{0}} u \mathrm{~d} x \mathrm{~d} \tau+\gamma\|v\|_{\infty}^{\mu_{1}, Q_{0}} \\
& \times\left(\iint_{Q_{0}} u^{\mu_{1}} \mathrm{~d} x \mathrm{~d} \tau\right)^{\mu_{2}}\left(\iint_{Q_{0}} v^{\mu^{\prime}} \mathrm{d} x \mathrm{~d} \tau\right)^{\mu_{1}}
\end{aligned}
$$

where $r>(N+2) / 2$, and $\mu_{1}, \mu_{2}$ have been defined in the statement. A similar argument applied to $(2.1 b)$ with $f=u^{q}$ and $\alpha=p$ gives

$$
\begin{aligned}
\|v\|_{\infty, Q_{\infty}} \leq & H f \int_{Q_{0}} v \mathrm{~d} x \mathrm{~d} \tau+\gamma\|u\|_{\infty}^{\sigma_{1}(s-1)} \\
& \times\left(\iint_{Q_{0}} v^{p} \mathrm{~d} x \mathrm{~d} \tau\right)^{\sigma_{2}}\left(\iint_{Q_{0}} u^{q} \mathrm{~d} x \mathrm{~d} \tau\right)^{\sigma_{1}},
\end{aligned}
$$

where $s>(N+2) / 2, \sigma_{1}$ has been defined above, and $\sigma_{2}=\left(1-\sigma_{1} s\right) / p$. Next we take advantage of $(2.32)$, (2.33) to obtain

$$
\begin{aligned}
\|u\|_{\infty} Q_{\infty} \leq & H \iint_{Q_{0}} u \mathrm{~d} x \mathrm{~d} \tau+\left(\iint_{Q_{0}} u^{u} \mathrm{~d} x \mathrm{~d} \tau\right)^{\mu_{2}} \\
& \times\left(\iint_{Q_{0}} v^{p} \mathrm{~d} x \mathrm{~d} \tau\right)^{\mu_{1}}\left(H \iint_{Q_{0}} v \mathrm{~d} x \mathrm{~d} \tau\right)^{p \mu_{1}(r-1)} \\
& +\gamma\|u\|_{\infty}^{\chi_{1}, Q_{\infty}}\left(\iint_{Q_{0}} u^{q} \mathrm{~d} x \mathrm{~d} \tau\right)^{x_{2}}\left(\iint_{Q_{0}} v^{p} \mathrm{~d} x \mathrm{~d} \tau\right)^{x}
\end{aligned}
$$

with $\chi_{1}, \chi_{2}, \chi_{3}$ as defined in the statement. It follows from easy calculations that, if $(2.30)$ holds, then $r, s>(N+2) / 2$ can be chosen so that $\chi_{1}<1$. We assume below that such a choice has been made. As a matter of fact, a rigorous derivation of $(2.34)$ requires of using suitable intermediate-size 
cylinders, as in the last part of Lemma 2.6. For ease of notation, though, we shall omit here and henceforth such auxiliary steps. Estimate (2.31a) follows by using Young's inequality to split the last term in (2.34). The analogous estimate for $v$ is obtained in a similar way.

Proof of Theorem 1. - We set $\rho^{2}=C(T-t)$ in (2.31). One readily sees that the quantity $H$ is bounded above by some positive constant. Moreover, one may use now (2.4) and (2.13) to obtain (after routine but tedious computations) that all three terms on the right hand side of $(2.31 \mathrm{a})$ provide the same bound $\gamma(T-t)^{-\frac{p+1}{p q-1}}$, and the estimate of $u$ follows. The bound for $v$ is obtained similarly.

Remark. - The local $L^{\infty}$ bounds given in this section actually hold for positive subsolutions of more general systems

$$
u_{t}-\operatorname{diva}(x, t, u, \nabla u)=v^{p}, \quad v_{t}-\operatorname{div} \mathbf{b}(x, t, v, \nabla v)=u^{q},
$$

provided $\mathbf{a}, \mathbf{b}$ satisfy standard structure assumptions. If $(u, v)$ is a solution of (2.35), the positivity requirement can be dropped, working separately with the positive and negative parts of $u, v$.

\section{A LIOUVILLE THEOREM}

This Section is devoted to the proof of Theorem 2. In what follows, we shall assume that

$$
1<p, q<\frac{N+2}{N-2} \text { if } N \geq 3 \quad \text { (any } p, q>1 \text { if } N=1,2 \text { ). }
$$

Let $(u(x, t), v(x, t))$ be a solution of (2.1) which blows up at $x=0, t=T$. We recall the auxiliary variables $\Phi, \Psi, y$ and $\tau$ introduced in (1.16). One readily checks that $\Phi$ and $\Psi$ satisfy

$$
\begin{aligned}
& \Phi_{\tau}=\Delta \Phi-\frac{1}{2} y \nabla \Phi+\Psi^{p}-\left(\frac{p+1}{p q-1}\right) \Phi, \\
& \Psi_{\tau}=\Delta \Psi-\frac{1}{2} y \nabla \Psi+\Phi^{q}-\left(\frac{q+1}{p q-1}\right) \Psi .
\end{aligned}
$$

Let $\Gamma, \gamma$ be the constants defined as the positive solutions of the equations

$$
\gamma^{p}=\Gamma \frac{p+1}{p q-1}, \quad \Gamma^{q}=\gamma \frac{q+1}{p q-1} .
$$

A crucial role in the proof of Theorem 2 is played by the following Vol. $14, n^{\circ} 1-1997$ 
Proposition 3.1. - Assume that there exists $K>0$ such that

$$
0 \leq \Phi \leq K, \quad 0 \leq \Psi \leq K, \quad \text { for }-\infty<\tau<\infty .
$$

Then, for any $p_{0} \in(1,(N+2) /(N-2))$ when $N \geq 3$ (any $p_{0}>1$ if $N=1,2)$ and any $\varepsilon>0$ there exists $\delta>0$ such that, if $\left|p-p_{0}\right|+\left|q-p_{0}\right|<\delta$, one of the following cases must necessarily occur

$$
\sup _{-\infty<\tau<\infty}(\|\Phi(\cdot, \tau)-\Gamma\|+\|\Psi(\cdot, \tau)-\gamma\|) \leq \varepsilon
$$

or

$$
\Phi \equiv \Psi \equiv 0
$$

or

$$
\limsup _{\tau \rightarrow-\infty}(\|\Phi(\cdot, \tau)-\Gamma\|+\|\Psi(\cdot, \tau)-\gamma\|) \leq \varepsilon
$$

and

$$
\lim _{\tau \rightarrow \infty}(\|\Phi(\cdot, \tau)\|+\|\Psi(\cdot, \tau)\|)=0 .
$$

To prove the Proposition, we proceed in several steps. Consider first the scalar equation

$$
\Phi_{\tau}=\Delta \Phi-\frac{1}{2} y \nabla \Phi+\Phi^{p}-\frac{\Phi}{p-1}, \quad \text { in } \mathbf{R}^{N} .
$$

Then there holds

LEMMA 3.2. - Let $\Phi(y, \tau)$ be a nonnegative and bounded solution of (3.5) which is defined for all $\tau \in(-\infty, \infty)$. Then one of the following possibilities must occur

$$
\Phi(y, \tau) \equiv(p-1)^{-\frac{1}{p-1}}
$$

or

$$
\Phi(y, \tau) \equiv 0
$$

or

$\Phi(y, \tau) \rightarrow(p-1)^{-\frac{1}{p-1}}, \quad$ as $\tau \rightarrow-\infty, \quad$ and $\Phi(y, \tau) \rightarrow 0, \quad$ as $\tau \rightarrow \infty$.

Remark. - It is worth pointing out that (3.6c) actually takes place for the explicit solutions

$$
\Phi_{k}(y, \tau) \equiv \Phi_{k}(\tau)=\left((p-1)+k e^{\tau}\right)^{-\frac{1}{p-1}}
$$

where $k$ is any positive constant. 
Proof of 3.2. - We take up an argument already used by Giga and Kohn in [19], and consider thus the functional

$$
E(\Phi)=\int_{\mathbf{K}^{N}}\left(\frac{|\nabla \Phi|^{2}}{2}+\frac{\Phi^{2}}{2(p-1)}-\frac{\Phi^{p+1}}{p+1}\right) e^{-|y|^{2} / 4} \mathrm{~d} y
$$

Notice that $E(\Phi(\cdot, \tau))<\infty$ for any $\tau \in(-\infty, \infty)$ by interior regularity theory for parabolic equations since $\Phi$ is globally bounded. A quick check reveals that

$$
\frac{\mathrm{d}}{\mathrm{d} \tau} E(\Phi(\cdot, \tau))=-\int_{\mathbf{R}^{N}} \Phi_{\tau}^{2} e^{-|y|^{2} / 4} \mathrm{~d} y .
$$

Consider now the elliptic counterpart of (3.5), i.e.,

$$
\Delta \Phi-\frac{1}{2} y \nabla \Phi+\Phi^{p}-\frac{\Phi}{p-1}=0, \quad \text { for } y \in \mathbf{R}^{N} .
$$

Arguing as in [19], it then follows that

$$
\int_{-\infty}^{+\infty} \mathrm{d} \tau \int_{\mathbf{R}^{N}} \Phi_{\tau}^{2} e^{-|y|^{2} / 4} \mathrm{~d} y<\infty
$$

and

$$
\Phi(y, \tau) \rightarrow \Phi^{+}(y) \quad \text { as } \tau \rightarrow \infty, \quad \Phi(y, \tau) \rightarrow \Phi^{-}(y) \quad \text { as } \tau \rightarrow-\infty,
$$

where convergence is uniform on compact sets $|y| \leq R<\infty$, and $\Phi^{+}, \Phi^{-}$, are global, nonnegative and bounded solutions of (3.9).

As recalled in [19], it follows from the results in [18] that for $1<p<(N+2) /(N-2)$ we must have

$$
\Phi^{ \pm} \equiv 0, \quad \text { or } \quad \Phi^{ \pm} \equiv(p-1)^{-\frac{1}{p-1}}
$$

If $\Phi^{-}(y)=0$, then $\Phi(y, \tau)=0$ for all $\tau$ by (3.8), and (3.6b) holds. If $\Phi^{+}(y)=(p-1)^{-\frac{1}{p-1}}$, we observe that

$$
E\left(\Phi^{+}\right)=(p-1)^{-\frac{p+1}{p-1}}\left(\frac{1}{2}-\frac{1}{p+1}\right) \int_{\mathbf{R}^{N}} e^{-|y|^{2} / 4} \mathrm{~d} y>0
$$

whence $E\left(\Phi^{-}\right)=E\left(\Phi^{+}\right)>0$ and (3.6a) holds in this case. Finally, (3.6c) corresponds to the only remaining admissible situation, namely $\Phi^{-}(y)=(p-1)^{-\frac{1}{p-1}}$, and $\Phi^{+}(y)=0$.

Vol. $14, n^{\circ} 1-1997$. 
As a next step we show

LEMma 3.3. - Let $\left\{p_{j}\right\},\left\{q_{j}\right\}$ be sequences such that

$$
\lim _{j \rightarrow \infty} p_{j}=\lim _{j \rightarrow \infty} q_{j}=p
$$

where $1<p<(N+2) /(N-2)$ if $N \geq 3$, and $p>1$ if $N=1$, 2. Let $\left(\Phi_{j}, \Psi_{j}\right)$ denote the corresponding solutions of $(3.2)$ with $(p, q)$ replaced by $\left(p_{j}, q_{j}\right)$ there, and assume that (3.3) holds for any pair $\left(\Phi_{j}, \Psi_{j}\right)$. Then there exists a subsequence (also labelled by j) such that

$$
\lim _{j \rightarrow \infty} \Phi_{j}=\lim _{j \rightarrow \infty} \Psi_{j}=\Phi^{*}
$$

where $\Phi^{*}$ solves (3.5), and convergence is uniform on compact subsets of $\mathbf{R}^{N} \times \mathbf{R}$.

Proof. - It follows from (3.3) and a standard compactness argument that there exists a subsequence $\left(\Phi_{j}, \Psi_{j}\right)$ which converges as $j \rightarrow \infty$ on compact subsets of $\mathbf{R}^{N} \times \mathbf{R}$ towards a solution of the system

$$
\begin{aligned}
& \Phi_{\tau}=\Delta \Phi-\frac{1}{2} y \nabla \Phi+\Psi^{p}-\frac{\Phi}{p-1} \\
& \Psi_{\tau}=\Delta \Psi-\frac{1}{2} y \nabla \Psi+\Phi^{p}-\frac{\Psi}{p-1}
\end{aligned}
$$

We now claim that

$$
\Phi \equiv \Psi
$$

To show (3.12), we set $Z=\Phi-\Psi$, subtract both equations in (3.11) and use Kato's inequality to obtain

$$
(|Z|)_{\tau} \leq \Delta(|Z|)-\frac{1}{2} y \nabla(|Z|)-\frac{|Z|}{p-1} .
$$

Since $|Z| \leq K$ by assumption, we readily see that

$$
|Z(y, \tau)| \leq K e^{-\frac{\tau+\tau_{u}}{p-1}}, \quad \text { for any } \tau_{0}<\tau<\infty .
$$

Letting now $\tau_{0} \rightarrow-\infty$, (3.12) follows and the proof is concluded.

To proceed further, we now prove

Lemma 3.4. - Assume now that the hypotheses in Proposition 3.1 are satisfied. There exists $\varepsilon>0$ small enough such that, if for some $\tau_{0} \in \mathbf{R}$ 


$$
\left\|\Phi\left(\cdot, \tau_{0}\right)\right\|+\left\|\Psi\left(\cdot, \tau_{0}\right)\right\| \leq \varepsilon
$$

then one has that, setting $\sigma=\min (p, q)$,

$$
\begin{aligned}
& \mid \Phi(\cdot, \tau)\|+\| \Psi(\cdot, \tau) \| \leq C \varepsilon e^{-\frac{\sigma+1}{p q-1}\left(\tau-\tau_{0}\right)}, \\
& \text { for some } C>0 \text { and any } \tau \geq \tau_{0}+C / 2 .
\end{aligned}
$$

Proof. - Let us denote by $S_{0}(\tau)$ the semigroup associated to the differential operator $A_{0} \equiv \Delta-\frac{1}{2} y \nabla$. Taking advantage of (3.3) and Kato's inequality, one easily sees that

$$
(|\Phi|)_{\tau} \leq \Delta(|\Phi|)-\frac{1}{2} y \nabla(|\Phi|)+\alpha(|\Phi|+|\Psi|)
$$

for some $\alpha=\alpha(K, p, q)>0$. Since $|\Psi|$ satisfies a similar equation, it turns out that

$$
(|\Phi|+|\Psi|)_{\tau} \leq \Delta(|\Phi|+|\Psi|)-\frac{1}{2} y \nabla(|\Phi|+|\Psi|)+\alpha(|\Phi|+|\Psi|) .
$$

Hence

$$
|\Phi(\cdot, \tau)|+|\Psi(\cdot, \tau)| \leq e^{\alpha\left(\tau-\tau_{0}\right)} S_{0}\left(\tau-\tau_{0}\right)\left(\left|\Phi\left(\cdot, \tau_{0}\right)\right|+\left|\Psi\left(\cdot, \tau_{0}\right)\right|\right) .
$$

In view of (3.15) and the delayed regularizing effect which holds for operator $A_{0}$ ( $c f[26]$ ), one has that, for any $r>1$ there exists $C_{0}>0$ and $L>0$ so that

$$
\begin{aligned}
& \|\Phi(\cdot, \tau)\|_{r, w}+\|\Psi(\cdot, \tau)\|_{r, u} \leq C_{0}\left(\left\|\Phi\left(\cdot, \tau_{0}\right)\right\|+\left\|\Psi\left(\cdot, \tau_{0}\right)\right\|\right), \\
& \text { whenever } L / 2 \leq \tau-\tau_{0} \leq L
\end{aligned}
$$

where

$$
\|h\|_{r, w}^{r}=\int_{\mathbf{R}^{N}}|h(s)|^{r} e^{-|s|^{2} / 4} \mathrm{~d} s,
$$

this last definition being meant for functions $h$ for which the right hand side above converges. Recalling (3.13), we may now use variation of constants formula in (3.2), and take advantage of (3.15), (3.16) to obtain that

$$
\begin{aligned}
& \|\Phi(\cdot, \tau)\|_{r, w}+\|\Psi(\cdot, \tau)\|_{r, w} \leq \varepsilon M e^{-\frac{\sigma+1}{p q-1}\left(\tau-\tau_{0}\right)}, \\
& \text { for some } M>0 \text { and } \sigma=\min (p, q), \text { provided that } L / 2 \leq \tau-\tau_{0} \leq L
\end{aligned}
$$


We next use variation of constants formula in (3.2) to obtain that

$$
\|\Phi(\cdot, \tau)\|+\|\Psi(\cdot, \tau)\| \leq F_{1}\left(\tau ; \tau_{0}, L\right)+F_{2}\left(\tau ; \tau_{0}, L\right)
$$

for $L / 2 \leq \tau-\tau_{0} \leq L$, where

$$
\begin{aligned}
& \left\|F_{1}\left(\tau ; \tau_{0}, L\right)\right\|=e^{-\frac{\sigma+1}{p q-1}\left(\tau-\left(\tau_{0}+\frac{L}{2}\right)\right)} \| S_{0}\left(\tau-\left(\tau_{0}+\frac{L}{2}\right)\right) \\
& \times\left(\Phi\left(\cdot \tau-\left(\tau_{0}+\frac{L}{2}\right)\right)+\left(\Psi\left(, \tau-\left(\tau_{0}+\frac{L}{2}\right)\right)\right) \|\right.
\end{aligned}
$$

and

$$
\left\|F_{2}\left(\tau ; \tau_{0}, L\right)\right\|=\int_{\tau_{0}+\frac{L}{2}}^{\tau} e^{-\frac{\sigma+1}{p q-1}(\tau-s)}\left\|S_{0}(\tau-s)\left(\Psi(\cdot, s)^{p}+\Phi(\cdot, s)^{q}\right)\right\| \mathrm{d} s .
$$

Since $\left\|S_{0}(\tau-s) f(\cdot, s)\right\| \leq C\|f(\cdot, s)\|$, it follows from (3.15) that $\left\|\Phi\left(\cdot, \tau_{0}+\frac{L}{2}\right)+\Psi\left(\cdot, \tau_{0}+\frac{L}{2}\right)\right\| \leq \varepsilon e^{\alpha L / 2}$. Hence

$$
\left\|F_{1}\left(\tau ; \tau_{0}, L\right)\right\| \leq \varepsilon C_{1} e^{-\frac{\sigma+1}{p q-1}\left(\tau-\left(\tau_{0}+\frac{L}{2}\right)\right)}, \text { for some } C_{1}>0
$$

To bound $F_{2}$, we first observe that

$$
\left\|S_{0}(\tau-s) f(\cdot, s)^{p}\right\| \leq C\left\|f(\cdot, s)^{p}\right\| \leq C\|f(\cdot, s)\|_{2 p, w}^{p} .
$$

We then make use of (3.17) (or rather of a variant of it, since we set $r=2 q$ in $\|\Phi(\cdot, \tau)\|_{r, w}$ and $r=2 p$ in $\left.\|\Psi(\cdot, \tau)\|_{r, w}\right)$ to obtain that

$$
\left\|F_{2}\left(\tau ; \tau_{0}, L\right)\right\| \leq \varepsilon^{\sigma} M^{\mu} e^{-\frac{\sigma+1}{p q-1}\left(\tau-\tau_{0}+\frac{L}{2}\right)}, \quad \text { for some } M>1
$$

where $\mu=\max (p, q)$. It then follows from (3.19) that

$$
\begin{gathered}
\|\Phi(\cdot, \tau)\|+\|\Psi(\cdot, \tau)\| \leq\left(\varepsilon C_{1}+\varepsilon^{\sigma} M^{\mu}\right) e^{-\frac{\sigma+1}{p q-1}\left(\tau-\tau_{0}+\frac{L}{2}\right)} \\
\text { provided that } \tau_{0}+L / 2 \leq \tau \leq \tau_{0}+L
\end{gathered}
$$

Recalling ( $(3.16 a)$, one then has that

$$
\begin{aligned}
& \|\Phi(\cdot, \tau)\|_{r, w}+\|\Psi(\cdot, \tau)\|_{r, w} \leq C_{0}\left(\varepsilon C_{1}+\varepsilon^{\sigma} M^{\mu}\right) \\
& \text { whenever } \tau_{0}+L \leq \tau \leq \tau_{0}+3 L / 2
\end{aligned}
$$

If we select now $\varepsilon>0$ such that $\varepsilon C_{1}+\varepsilon^{\sigma} M^{\mu} \leq 2 \varepsilon C_{1}$ and $M>0$ in (3.17) so that $M>2 C_{0} C_{1}$, we can repeat the previous argument with $\tau_{0}$ replaced 
by $\tau_{0}+L / 2$ to obtain (3.20), this time in the interval $\tau_{0}+L \leq \tau \leq \tau_{0}+3 L / 2$. Iterating this procedure, the result follows.

End of the Proof of Proposition 3.1. - Assume that (3.4a) fails to hold. Keeping to the notation in Lemma 3.3, there exists $\varepsilon>0$ and sequences $\left\{p_{j}\right\},\left\{q_{j}\right\}$ and $\left\{\tau_{j}\right\}$ such that $\lim _{j \rightarrow \infty} p_{j}=\lim _{j \rightarrow \infty} q_{j}=p_{0}$, $1<p_{0}<(N+2) /(N-2)$ if $N \geq 3\left(p_{0}>1\right.$ if $\left.N=1,2\right)$, and

$$
\left\|\Phi_{j}\left(\cdot, \tau_{j}\right)-\Gamma_{j}\right\|+\left\|\Psi_{j}\left(\cdot, \tau_{j}\right)-\gamma_{j}\right\|>\varepsilon,
$$

where $\Gamma_{j}, \gamma_{j}$ are given by (1.18) with $p, q$ replaced by $p_{j}$ and $q_{j}$ there. Let us continue to denote by $\Phi_{j}(y, \tau), \Psi_{j}(y, \tau)$ the functions given respectively by $\Phi_{j}\left(y, \tau+\tau_{j}\right)$ and $\Psi_{j}\left(y, \tau+\tau_{j}\right)$. Then we have that

$$
\left\|\Phi_{j}(\cdot, 0)-\Gamma_{j}\right\|+\left\|\Psi_{j}(\cdot, 0)-\gamma_{j}\right\|>\varepsilon .
$$

By Lemma 3.3 it follows that a subsequence (still denoted by $\left\{\Phi_{j}\right\},\left\{\Psi_{j}\right\}$ ) should converge to a solution $\Phi^{*}(y, \tau)$ of (3.5) which is defined for all $\tau \in(-\infty, \infty)$. These last solutions have been classified in Lemma 3.2. By assumption (3.6a) cannot hold, so that either (3.6b) or (3.6c) should occur. In either case, one has that for some $\tau_{0},\left\|\Phi_{j}\left(\cdot, \tau_{0}\right)\right\|+\left\|\Psi_{j}\left(\cdot, \tau_{0}\right)\right\|$ becomes small enough, and then Lemma 3.4 yields $\left\|\Phi_{j}(\cdot, \tau)\right\|+\left\|\Psi_{j}(\cdot, \tau)\right\| \rightarrow 0$ as $\tau \rightarrow \infty$. Suppose now that the limit function $\Phi^{*}(y, \tau)$ is not identically zero. We then claim that

$$
\left\|\Phi_{j}(\cdot, \tau)-\Gamma_{j}\right\|+\left\|\Psi_{j}(\cdot, \tau)-\gamma_{j}\right\| \leq C, \quad \text { as } \tau \rightarrow-\infty,
$$

where $C=C\left(p_{j}, q_{j}\right) \rightarrow 0$ as $j \rightarrow \infty$. The proof of this last fact follows again by contradiction, since $\Phi^{*}$ necessarily is either a $S$-shaped function as that in $(3.6 c)$, or zero. This concludes the proof.

We now turn our attention to the situation where (3.4a) holds. Our aim consists in proving that, in such case

$$
\Phi \equiv \Gamma, \quad \Psi \equiv \gamma
$$

To this end, we linearise around $(\Gamma, \gamma)$ by setting

$$
\varphi=\Phi-\Gamma, \quad \psi=\Psi-\gamma
$$

One then obtains

$$
\begin{aligned}
\varphi_{\tau} & =\Delta \varphi-\frac{1}{2} y \nabla \varphi+(\gamma+\psi)^{p}-\frac{p+1}{p q-1}(\Gamma+\varphi) \\
& =\Delta \varphi-\frac{1}{2} y \nabla \varphi-\left(\frac{p+1}{p q-1}\right) \varphi+p \gamma^{p-1} \psi+f_{1}(\psi) \\
& \equiv A_{1} \varphi+p \gamma^{p-1} \psi+f_{1}(\psi)
\end{aligned}
$$


where $f_{1}(\psi)=O\left(\psi^{2}\right)$ as $\psi \rightarrow 0$. In a similar way, we obtain

$$
\begin{aligned}
\psi_{\tau} & =\Delta \psi-\frac{1}{2} y \nabla \psi-\left(\frac{q+1}{p q-1}\right) \psi+q \Gamma^{q-1} \varphi+f_{2}(\varphi) \\
& \equiv A_{2} \psi+q \Gamma^{q-1} \varphi+f_{2}(\varphi)
\end{aligned}
$$

where $f_{2}(\varphi)=O\left(\varphi^{2}\right)$ as $\varphi \rightarrow 0$. As a next step we define a function space $V=\left\{\left(\begin{array}{l}f \\ g\end{array}\right) \mid f, g \in L_{w}^{2}\left(\mathbf{R}^{N}\right)\right\}$, and a linear operator $A: V \rightarrow V$ with domain $D(A)=H_{w}^{2}\left(\mathbf{R}^{N}\right) \times H_{w}^{2}\left(\mathbf{R}^{N}\right)$, given by

$$
A=\left(\begin{array}{cc}
A_{1} & p \gamma^{p-1} I \\
q \Gamma^{q-1} I & A_{2}
\end{array}\right)
$$

where, as usual, $I$ denotes the identity operator. Notice that if $p \neq q$, $q \Gamma^{q-1} \neq p \gamma^{p-1}$, and in such case $A$ is not self-adjoint in the space $V$ endowed with the natural scalar product

$$
\left\langle\left(\begin{array}{l}
f \\
g
\end{array}\right),\left(\begin{array}{l}
\bar{f} \\
\bar{g}
\end{array}\right)\right\rangle=\int_{\mathbf{R}^{N}}(f \bar{f}+g \bar{g}) e^{-|y|^{2} / 4} \mathrm{~d} y
$$

This lack of self-adjointness translates into some modifications in our forthcoming approach with respect to that corresponding to the scalar case (cf [26], [27]). We shall next sketch the relevant details. We start with operator $A_{0} \equiv \Delta-\frac{1}{2} y \nabla$, already considered in Lemma 3.4. By standard results (which have also been recalled in the Introduction), $A_{0}$ is self-adjoint in $L_{w}^{2}\left(\mathbf{R}^{N}\right)$. Moreover, if we denote elements in $\mathbf{R}^{N}$ by $\alpha=\left(\alpha_{1}, \ldots, \alpha_{N}\right)$, the spectrum of $A_{0}$ consists of values $-|\alpha| / 2$, where $\alpha_{i}$ is a nonnegative integer for any $i=1,2, \ldots, N$, and $|\alpha|=\alpha_{1}+\cdots+\alpha_{N}=0$, $1,2, \ldots$. The corresponding eigenfunctions are given by $c_{\alpha} H_{\alpha}(y)=$ $c_{\alpha_{1}} H_{\alpha_{1}}\left(y_{1}\right) \cdots c_{\alpha_{N}} H_{\alpha_{N}}\left(y_{N^{*}}\right)$, where $H_{n}(s)=\tilde{H}_{n}(s / 2), \hat{H}_{n}$ being the standard $n^{\text {th }}$-Hermite polynomial. The constant $c_{\alpha}=\left(c_{\alpha_{1}}, \ldots, c_{\alpha_{N}}\right)$ is selected so that $\left|c_{\alpha}\right|^{2}\left\langle H_{\alpha}, H_{\alpha}\right\rangle=1$. The family $\left\{H_{\alpha \alpha}\right\}$ constitutes an orthonormal basis in $L_{w}^{2}\left(\mathbf{R}^{N}\right)$. This last space is isomorphic to the space of sequences $\ell^{2}=\left\{\left.\left(x_{\alpha}\right)\left|\sum_{\alpha}\right| x_{\alpha}\right|^{2}<\infty ; \alpha=\left(\alpha_{1}, \ldots, \alpha_{N}\right)\right.$ as above $\}$. As it is well known, this isomorphism is given by the mapping $f \mapsto\left\{a_{\alpha}\right\}$, where

$$
f=\sum_{\alpha}\left\langle f, H_{\kappa}\right\rangle H_{\alpha} \equiv \sum_{\alpha} a_{\alpha} H_{\alpha} .
$$

This correspondence is extended up to an isomorphism between $V$ and $\ell^{2}\left(\mathbf{C}^{2}\right)=\left\{\left(a_{\alpha}, b_{\alpha}\right) \in \mathbf{C}^{2} \mid \sum_{\alpha}\left(\left|a_{\alpha}\right|^{2}+\left|b_{\alpha}\right|^{2}\right)<\infty, \alpha\right.$ as before $\}$ in a 
natural manner. Namely, denoting transposition by * for ease of notation, we associate to every pair $(f, g)^{*} \in V$ an element $\left(\left\{f_{\alpha}\right\},\left\{g_{\alpha}\right\}\right)^{*}$ in $\ell^{2}\left(\mathbf{C}^{2}\right)$ given by

$$
f_{\alpha}=\left\langle f \cdot H_{\alpha}\right\rangle, \quad g_{\alpha}=\left\langle g, H_{\alpha}\right\rangle ; \quad \alpha=\left(\alpha_{1}, \ldots \alpha_{\gamma}\right) .|\alpha|=0,1,2, \ldots
$$

so that the action of the operator $A$ given in (3.23) on the space $\ell^{2}\left(\mathbf{C}^{2}\right)$ is described as follows

$$
\left(A\left(\begin{array}{l}
u \\
v
\end{array}\right)\right)_{\alpha}=\left(\begin{array}{cc}
-\frac{|\alpha|}{2}-\frac{p+1}{p q-1} & p \gamma^{p-1} \\
q \Gamma^{q-1} & -\frac{|\alpha|}{2}-\frac{q+1}{p q-1}
\end{array}\right)\left(\begin{array}{l}
u_{\alpha} \\
v_{\alpha}
\end{array}\right) .
$$

Set now

$$
M=\left(\begin{array}{cc}
-\frac{p+1}{p q-1} & p \gamma^{p-1} \\
q \Gamma^{q-1} & -\frac{q+1}{p q-1}
\end{array}\right)
$$

A straightforward computation reveals that $M$ has eigenvalues $\lambda^{+}, \lambda^{-}$ given by

$$
\lambda^{+}=1, \quad \lambda^{-}=-\frac{(p+1)(q+1)}{p q-1} .
$$

It is therefore possible to select $u_{+}, u_{-}$such that

$$
M u_{+}=u_{+} . \quad M u_{-}=\lambda^{-} u_{-}, \quad\left|u_{+}\right|=\left|u_{-}\right|=1 .
$$

Notice that $u_{+}$and $u_{-}$are parallel respectively to

$$
\left(1, \frac{q+1}{p q-1} \gamma^{1-p}\right)^{*}: \quad\left(1,-\frac{q(p+1)}{p q-1}\left(p \gamma^{p-1}\right)^{-1}\right)^{*} \text {. }
$$

Bearing in mind the previous remarks, we now write

$$
(f, g)^{*}=\sum_{\alpha} a_{\alpha}^{+} H_{\alpha} u_{+}+\sum_{\alpha} a_{\alpha}^{-} H_{\alpha} u_{-}
$$

for any pair $(f, g)^{*} \in V$. A word of caution is required, however, concerning (3.26a), since $\left\{H_{\alpha} u_{+}\right\} \cup\left\{H_{\alpha} u_{-}\right\}$is not an orthonormal basis in $V$ (this Vol. $14, n^{\circ} 1.1997$. 
follows from the fact that $u_{+}$and $u_{-}$are not orthogonal). Nevertheless, we may define subspaces $V_{+}$in the form

$$
V_{ \pm}=\left\{\left(a_{\alpha}, b_{\alpha}\right)^{*}=c_{\alpha} u_{ \pm} ; \sum_{\alpha}\left|c_{\alpha}\right|^{2}<\infty\right\}
$$

and we still have that

$$
V=V_{+} \oplus V_{-}, \quad \text { but } V_{+} \text {is not orthogonal to } V_{-} \text {. }
$$

Summing up, we have obtained that the spectrum of operator $A$ in (3.23) is given by

$$
\begin{gathered}
A\left(H_{\alpha} u_{+}\right)=\left(1-\frac{|\alpha|}{2}\right) H_{\alpha} u_{+}, \\
A\left(H_{\alpha} u_{-}\right)=-\left(\frac{|\alpha|}{2}+\frac{(p+1)(q+1)}{p q-1}\right) H_{\alpha} u_{-},
\end{gathered}
$$

where, as before, $\alpha=\left(\alpha_{1}, \ldots, \alpha_{N}\right)$ and for any $i$ with $1 \leq i \leq N, \alpha_{i}$ is a nonnegative (possibly zero) integer. There are two positive eigenvalues: $\lambda_{1}=1$, to which it corresponds the eigenfunction $H_{0} u_{+}$, and $\lambda_{2}=1 / 2$. In this last case, there are $N$ eigenfunctions, given by $H_{c x} u_{+}$, where $\alpha$ runs through the unit vectors $(1,0, \ldots, 0), \ldots,(0, \ldots, 0,1)$. The eigenvalue $\lambda_{3}=0$ has in turn $N(N+1) / 2$ eigenfunctions of the form $H_{\alpha} u_{+}$, where the index $\alpha$ has either two coordinates equal to 1 or one coordinate equal to 2 , all others being zero. All remaining eigenvalues are negative.

At this juncture, a couple of remarks are in order. First, for any pair $(\varphi, \psi)^{*} \in V$ there holds

$$
c_{1} \sum_{\alpha}\left(\left|a_{\alpha}^{+}\right|^{2}+\left|a_{\alpha}^{-}\right|^{2}\right) \leq\left\langle(\varphi, \psi)^{*},(\varphi, \psi)^{*}\right\rangle \leq c_{2} \sum_{\alpha}\left(\left|a_{\alpha}^{+}\right|^{2}+\left|a_{\alpha}^{-}\right|^{2}\right)
$$

for some positive constants $c_{1}$ and $c_{2}$. We shall henceforth use the notation

$$
\begin{aligned}
v(y, \tau) & =(\varphi(y, \tau), \psi(y, \tau))^{*}, \\
\|v(\cdot, \tau)\| & =\left\|(\varphi(\cdot, \tau), \psi(\cdot, \tau))^{*}\right\| .
\end{aligned}
$$

Second, we should stress that our functional frame allows us to make use of the delayed regularizing effects introduced in [21] and [26] to study the scalar case. These will he used in deriving our next result, which reads as follows 
Lemma 3.5. - For any $\sigma>0$ there exists $\varepsilon>0$ such that, if (3.4a) holds, then

$$
\sum_{|\alpha| \leq 1}\left|a_{\alpha}^{+}\right| \leq \sigma\|v(\cdot, \tau)\|, \quad \text { for }-\infty<\tau<\infty
$$

where $v(\cdot, \tau)$ is given in (3.29).

Proof. - We shall argue by contradiction. Assume then that there exists a real $\vec{\tau}$ and $\sigma>0$ so that

$$
\sum_{|\alpha| \leq 1}\left|a_{\alpha}^{+}(\tilde{\tau})\right|>\sigma\|v(\cdot, \bar{\tau})\|
$$

regardless of how small $\varepsilon>0$ in (3.4a) is. For the ease of notation, we shall assume $\sigma=1$ in what follows. In view of our previous discussion, we have that

$$
v_{\tau}=A v+f(v)
$$

where $A$ is given in (3.22), (3.23), and $f(v)=O\left(v^{2}\right)$ as $v \rightarrow 0$. If we denote now by $S(\tau)$ the semigroup associated to $A$, we may represent solutions of (3.32) in the form

$$
v(\cdot, \tau)=S(\tau-\bar{\tau}) v(\cdot, \bar{\tau})+\int_{\bar{\tau}}^{\tau} S(\tau-s) f(v(\cdot, s)) \mathrm{d} s, \quad \text { for } \tau>\bar{\tau} .
$$

Let $r>1$ be large (but fixed). Then there exists $L>0$ such that

$$
\|v(\cdot, s)\|_{r, w} \leq C\|v(\cdot, \bar{\tau})\|, \quad \text { for } s \geq \bar{\tau}+L
$$

Take now $\tau$ so that $2 L \leq \tau-\bar{\tau} \leq 3 L$, and split the second term on the right of (3.33) as follows

$$
\int_{\bar{\tau}}^{\tau} S(\tau-s) f(v(\cdot, s)) \mathrm{d} s=\int_{\bar{\tau}}^{\tau-L} \cdots+\int_{\tau-L}^{\tau} \cdots \equiv I_{1}+I_{2} .
$$

Using (3.31) and (3.34), we readily see that

$$
\left\|I_{2}\right\| \leq c\left(\sum_{|\alpha| \leq 1}\left|a_{\alpha}^{+}(\bar{\tau})\right|\right)^{2}
$$

where here and in what follows $C$ denotes a positive generic constant. As to $I_{1}$, we notice that the value of $L$ is determined by our choice of $r$ in Vol. $14, n^{\circ} 1-1997$. 
(3.34), and this in turn has been already fixed to derive the bound (3.36). It may therefore happen that (3.36) does not continue to hold when we replace $I_{2}$ by $I_{1}$ there. In such case, we argue as follows. Select $\delta>0$ so that $f(v)=O\left(v^{1+\delta}\right)$ as $v \rightarrow 0$, where $\delta$ is small enough so as to have

$$
\left\|v(\cdot, s)^{1+\delta}\right\|_{\frac{2}{1+\delta} \cdot u} \leq C\left(\sum_{|\alpha| \leq 1}\left|a_{a}^{+}(\bar{\tau})\right|\right)^{1+\phi}, \quad \text { for } s \geq \bar{\tau}+L .
$$

We then have that

$$
\left\|I_{1}\right\| \leq c\left(\sum_{|\alpha| \leq 1}\left|a_{\alpha}^{+}(\bar{\tau})\right|\right)^{1+\delta}
$$

whence, using (3.33)

$$
\begin{aligned}
\sum_{|\alpha| \leq 1}\left|a_{\alpha}^{+}(\tau)\right| \geq & \sum_{|\alpha| \leq 1}\left|a_{\alpha}^{+}(\bar{\tau})\right| e^{(\tau-\bar{\tau}) / 2} \\
& -C\left[\left(\sum_{|\alpha| \leq 1}\left|a_{\alpha}^{+}(\bar{\tau})\right|\right)^{2}+\left(\sum_{|\alpha| \leq 1}\left|a_{\alpha}^{+}(\bar{\tau})\right|\right)^{1+\delta}\right] .
\end{aligned}
$$

Hence

$$
\begin{aligned}
& \sum_{|\alpha| \leq 1}\left|a_{\alpha}^{+}(\tau)\right| \geq \sum_{|\alpha| \leq 1}\left|a_{\alpha}^{+}(\bar{\tau})\right| . \quad \text { for } 2 L \leq \tau-\bar{\tau} \leq 3 L, \\
& \text { regardless of how small } \varepsilon>0 \text { in (3.4a) is, }
\end{aligned}
$$

whereas on the other hand

$$
\|v(\cdot, \tau)\| \leq C\|v(\cdot, \bar{\tau})\|, \quad \text { for } 2 L \leq \tau-\bar{\tau} \leq 3 L .
$$

We now write $v=v_{+}+v_{-}$with $v_{+} \in V_{+}$(resp. $v_{-} \in V_{-}$), where $V_{+}$, $V_{-}$are given in (3.26). We also set

$$
w_{+}=v_{+}-\sum_{|\alpha| \leq 1} H_{\alpha} u_{+}\left\langle H_{\alpha} u_{+}, v_{+}\right\rangle
$$

and define

$$
T(\tau)=\sum_{|\alpha| \leq 1}\left|a_{\alpha}^{+}(\tau)\right|^{2}\left(\left\|w_{+}(\cdot, \tau)\right\|^{2}+\left\|v_{-}(\cdot, \tau)\right\|^{2}\right)^{-1} .
$$


Clearly,

$$
T(\tau) \geq \theta_{1}>0, \quad \text { for } \bar{\tau}+2 L \leq \tau \leq \bar{\tau}+3 L, \quad \text { and some } \theta_{1}>0 . \quad \text { (3.42) }
$$

We now compute

$$
\begin{aligned}
\frac{\mathrm{d} T}{\mathrm{~d} \tau}= & 2 \sum_{|\alpha| \leq 1} a_{\alpha}^{+}\left(a_{\alpha}^{+}\right)\left(\left\|w_{+}(\cdot, \tau)\right\|^{2}+\left\|v_{-}(\cdot, \tau)\right\|^{2}\right)^{-1} \\
& -2 \sum_{|\alpha| \leq 1}\left(a_{\alpha}^{+}(\tau)\right)^{2}\left(\left\|w_{+}(\cdot, \tau)\right\|^{2}+\left\|v_{-}(\cdot, \tau)\right\|^{2}\right)^{-2} \\
& \times\left(\left\langle w_{+},\left(w_{+}\right)_{\tau}\right\rangle+\left\langle v_{-}\left(v_{-}\right)_{\tau}\right\rangle\right)
\end{aligned}
$$

Notice that operator $A$ commutes with the decomposition $V=V_{+} \oplus V$. Therefore, denoting by $P_{+}$(resp. $P_{-}$) the projection operator on $V_{+}$(resp. on $V_{-.}$), equation (3.32) gives raise to

$$
\begin{aligned}
& \left(v_{+}\right)_{\tau}=A\left(v_{+}\right)+P_{+}(f(v)) . \\
& \left(v_{-}\right)_{\tau}=A\left(v_{-}\right)+P_{-}(f(v)) .
\end{aligned}
$$

Let $Q$ be the projection operator from $V_{+}$onto the subspace orthogonal to the eigenfunctions $H_{\mathrm{a}} u_{+}$with $\alpha=0$ or $\alpha=(1,0, \ldots, 0), \ldots$, $\alpha=(0, \ldots, 0,1)$. Thus $w_{+}=Q v_{+}$, and

$$
\left(w_{+}\right)_{\tau}=A\left(w_{+}\right)+Q P_{+}(f(v)) .
$$

Using (3.43)-(3.45) we obtain

$$
\begin{aligned}
\frac{\mathrm{d} T}{\mathrm{~d} \tau}= & 2 \sum_{|\alpha| \leq 1}\left(1-\frac{|\alpha|}{2}\right)\left|a_{\alpha}^{+}\right|^{2}\left(\left\|w_{+}(\cdot, \tau)\right\|^{2}+\left\|v_{-}(\cdot, \tau)\right\|^{2}\right)^{-1} \\
& -2 \sum_{|\alpha| \leq 1}\left|a_{\alpha}^{+}\right|^{2}\left(\left\|w_{+}(\cdot, \tau)\right\|^{2}+\left\|v_{-}(\cdot, \tau)\right\|^{2}\right)^{-2} \\
& \times\left(\left\langle w_{+}, A w_{+}\right\rangle+\left\langle w_{+}, Q P_{+}(f(v))\right\rangle+\left\langle v_{-}, A v_{-}\right\rangle+\left\langle v_{-}, P_{-}(f(v))\right\rangle\right)
\end{aligned}
$$

As $\left\langle w_{+}, A w_{+}\right\rangle \leq 0$, and $\left\langle v_{-}, A v_{-}\right\rangle \leq-c\left\|v_{-}\right\|^{2}$, it then follows that

$$
\begin{aligned}
\frac{\mathrm{d} T}{\mathrm{~d} \tau} \geq & 2 \sum_{|\alpha \times| \leq 1}\left|a_{\alpha}^{+}\right|^{2}\left(\left\|w_{+}(\cdot, \tau)\right\|^{2}+\left\|v_{-}(\cdot, \tau)\right\|^{2}\right)^{-1} \\
& \times\left(\frac{1}{2}-\left(\left|\left\langle w_{+}, Q P_{+} f(v)\right\rangle\right|\right.\right. \\
& \left.\left.+\left|\left\langle v_{-}, P_{-} f(v)\right\rangle\right|\right)\left(\left\|w_{+}(\cdot \tau)\right\|^{2}+\left\|v_{-}(\cdot \tau)\right\|^{2}\right)^{-1}\right)
\end{aligned}
$$


We next remark that i), we have the estimate

$$
\left\|v(\cdot, \tau)^{3}\right\| \leq\|v(\cdot, \tau)\|_{6, u}^{3} \leq C\|v(\cdot, \tau-L)\|^{3},
$$

and ii), by (3.39) and (3.42), $\|v(\cdot, \tau-L)\| \leq C\|v(\cdot, \tau)\|$, where $C>0$ depends indeed on $L$ and $\theta_{1}$. Putting all together, we see that

$$
\left\|v(\cdot, \tau)^{3}\right\| \leq C\|v(\cdot, \tau)\|^{3}
$$

and hence

$$
\begin{aligned}
\frac{\mathrm{d} T}{\mathrm{~d} \tau} \geq & 2 \sum_{|\alpha| \leq 1}\left|a_{\alpha}^{+}\right|^{2}\left(\left\|w_{+}(\cdot, \tau)\right\|^{2}+\left\|v_{-}(\cdot, \tau)\right\|^{2}\right)^{-1} \\
& \times\left(\frac{1}{2}-C\|v(\cdot, \tau)\|^{3}\left(\left\|w_{+}(\cdot, \tau)\right\|^{2}+\left\|v_{-}(\cdot, \tau)\right\|^{2}\right)^{-1}\right) \\
\geq & 2 T(\tau)\left(\frac{1}{2}-C T(\tau)\|v(\cdot, \tau)\|\right) .
\end{aligned}
$$

Therefore, if $\varepsilon>0$ in $(3.4 a)$ is small enough, we obtain by a standard continuation argument that

$$
T(\tau) \geq \theta_{2}>0, \quad \text { for } \tau \geq \bar{\tau}+L
$$

We now claim that (3.46) cannot possibly hold. To wit, we notice that, by (3.32), one has that for $\tau \geq \bar{\tau}+L$

$$
\begin{aligned}
\frac{\partial}{\partial \tau} \sum_{|\alpha| \leq 1}\left|a_{\alpha}^{+}\right| & \geq \frac{1}{2} \sum_{|\alpha| \leq 1}\left|a_{\alpha}^{+}\right|-\left\|Q P_{1} f(v)\right\| \\
& \geq \frac{1}{2} \sum_{|\alpha| \leq 1}\left|a_{\alpha}^{+}\right|-C\|v\|^{2} \geq\left(\frac{1}{2}-C_{1} \varepsilon\right) \sum_{|\alpha| \leq 1}\left|a_{\alpha}^{+}\right|
\end{aligned}
$$

for some $C_{1}>0$, where we have made use of (3.4a) and (3.46) to derive the last inequality. Integrating the previous inequality in time we finally arrive at

$$
\sum_{|\alpha| \leq 1}\left|a_{\alpha}^{+}(\tau)\right| \geq \sum_{|\alpha| \leq 1}\left|a_{\alpha}^{+}(\bar{\tau}+L)\right| e^{\bar{\gamma}(\tau-\bar{\tau}-L)}, \quad \text { for } \tau \geq \bar{\tau}+L \text {, }
$$

where $\tilde{\gamma}=1 / 2-C_{1} \varepsilon$. I etting $\varepsilon \rightarrow \infty$, we obtain a contradiction with the fact that $v(\cdot, \tau)$ must be bounded. 
The final step is the proof of Theorem 2 is

Lemma 3.6. - For any $\sigma>0$ there exists $\varepsilon>0$ such that, if (3.4a) holds then

$$
\sum_{|\alpha|=2}\left|a_{\alpha}^{+}\right| \leq \sigma\|v(\cdot, \tau)\|, \quad-\infty<\tau<\infty .
$$

Proof. - It follows from the lines of that corresponding to our previous Lemma. Therefore, we shall merely sketch most of the arguments involved, to describe in more detail those parts where differences with respect to that proof appear. Let us write

$$
\begin{gathered}
v_{+}=\sum_{|\alpha| \leq 1} a_{\alpha}^{+} H_{\alpha} u_{+}+\sum_{|\alpha|=2} a_{\alpha}^{+} H_{\alpha} u_{+}+R_{+}(\tau), \\
T(\cdot, \tau)=\sum_{|\alpha|=2}\left|a_{\alpha}^{+}\right|^{2}\left(\left\|R_{+}(\cdot, \tau)\right\|^{2}+\left\|v_{-}(\cdot, \tau)\right\|^{2}\right)^{-1} .
\end{gathered}
$$

A straightforward modification of the arguments in Lemma 3.5 gives

$$
\begin{gathered}
\dot{T}(\tau) \geq 0, \quad \text { as far as (i) } T(\tau) \geq \theta>0, \text { for } \\
\text { some } \theta, \text { and (ii) } \varepsilon \text { in (3.4a) is small enough. }
\end{gathered}
$$

Fix now $\delta>0$. Then either

$$
\begin{gathered}
\sum_{|\alpha| \leq 2}\left|a_{\alpha}^{+}\left(\tau_{j}\right)\right| \geq \delta\left(\sum_{|\alpha| \leq 1}\left|a_{\alpha}^{+}\left(\tau_{j}\right)\right|+\left\|R_{+}\left(\cdot, \tau_{j}\right)\right\|+\left\|v_{-}\left(\cdot, \tau_{j}\right)\right\|\right), \\
\text { for some sequence }\left\{\tau_{j}\right\} \text { with } \lim _{j \rightarrow \infty} \tau_{j}=-\infty,
\end{gathered}
$$

or else

For any $M>0$ large enough, and any $\tau \leq-M$, there holds

$$
\sum_{|\alpha| \leq 2}\left|a_{\alpha}^{+}(\tau)\right|<\delta\left(\sum_{|\alpha| \leq 1}\left|a_{\alpha}^{+}(\tau)\right|+\left\|R_{+}(\cdot, \tau)\right\|+\left\|v_{-}(\cdot, \tau)\right\|\right) .
$$

Suppose now that (3.51) is satisfied. Then, using the equations for $v_{-}$and $R_{+}$(which are easily obtained from (3.32)) we derive

$$
\frac{\partial}{\partial \tau}\|v(\cdot, \tau)\| \leq-\ddot{\mu}\|v(\cdot, \tau)\|+O\left(\|v(\cdot, \tau-L)\|^{1+\eta}\right), \quad \text { for } \tau \leq-M,
$$


where $\tilde{\mu}>0$ and $\eta>0$ are (small) positive numbers. We may now use (3.4a) to see that, for any $\tau_{0}<\tau<-M$,

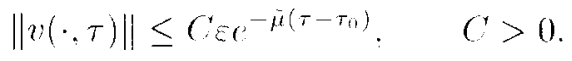

whence by letting $\tau_{0} \rightarrow-\infty$ we would obtain that $v(\cdot, \tau) \equiv 0$ for any $\tau \leq-M$, which in turn yields $v(\cdot \tau) \equiv 0$ for any $\tau \geq-M$ by a standard continuation argument.

It remains yet to consider the case where (3.50) occurs. i.e., when quadratic modes dominate in the expansion (3.26) with $(f, g)^{*}$ replaced there by $v=(\varphi, \psi)^{*}$. In this case, we may use a continuation argument involving $(3.49)$ to obtain that

$$
\sum_{|\alpha| \leq 2}\left|a_{\alpha}^{+}(\tau)\right| \geq \delta\left(\sum_{|\alpha| \leq 1}\left|a_{\alpha}^{+}(\tau)\right|+\left\|R_{+}(\cdot, \tau) \mid+\right\| v_{-}(\cdot, \tau) \|\right) . \quad \text { for any real } \%
$$

We now define

$$
\chi=\sum_{|\alpha|=2} a_{\alpha}(\tau) H_{\alpha} u_{+},
$$

and, as in [26], define the matrix $G=\left(G_{i j}\right), 1 \leq i, j \leq N$, whose entries are given by

$$
G_{i j}= \begin{cases}\sqrt{2}\left\langle\chi, H_{2}\left(y_{i}\right) u_{+}\right\rangle H_{0}^{N-1} . & \text { if } i=j \\ \left\langle\chi \cdot H_{1}\left(y_{i}\right) H_{1}\left(y_{j}\right) u_{+}\right\rangle H_{0}^{N-2} . & \text { if } i \neq j\end{cases}
$$

A minor modification of the arguments in [26] yields now

$$
\dot{G}=\nu G^{2}+\delta H(G) \text {. }
$$

where $\nu>0$, and $H(G)=O\left(\|G\|^{2}\right)$ for small $\|G\|$. Moreover, $G$ is symmetric, and its eigenvalues $\lambda_{k}(\tau)$ satisfy

$$
\dot{\lambda}_{k}=\theta_{p, q} \lambda_{k}^{2}+\delta O\left(\|\lambda\|^{2}\right)
$$

where $\|\lambda\|^{2}=\sum_{k} \lambda_{k}^{2}$, and coefficients $\theta_{p, q}$ are positive. The analysis of the O.D.E. system (3.53) is similar to that performed in [26], Section 3. It turns out that the eigenvalues $\left\{\lambda_{k}(\tau)\right\}$ satisfy (after a possible relabelling) that

$$
\begin{gathered}
\lambda_{k}(\tau)=-\frac{C}{\tau-\tau_{0}} . \quad \text { for } \tau \geq \tau_{0} . \quad k=1 \ldots \ell . \\
\lambda_{k}(\tau)=o\left(\frac{1}{\tau-\tau_{0}}\right) . \quad \text { for } \tau \geq \tau_{0}, \quad k=\ell+1, \ldots, N .
\end{gathered}
$$

Letting $\tau_{0} \rightarrow-\infty$, one then obtains that $\|\lambda\|=0$ and hence $\|G\|=0$, whence $\|v(\cdot, \tau)\|=0$.

Putting together Proposition 3.1, and Lemmata 3.2-3.6, the proof of Theorem 2 is now complete. 


\section{CLASSIFICATION OF SINGULARITIES. OCCURRENCE OF A PARTICULAR CLASS OF PROFILES}

In this Section we shall prove Theorems 3 and 4 in the Introduction.

\subsection{The proof of Theorem 3}

Our first result in this direction is

LEMMA 4.1. - Under the assumptions of Theorem 3, one has that

$$
\begin{aligned}
& \Phi(y, \tau) \rightarrow \Gamma, \quad \Psi(y, \tau) \rightarrow \gamma, \quad \text { as } \tau \rightarrow \infty, \\
& \text { uniformly on compact sets }|y| \leq R<\infty .
\end{aligned}
$$

Proof. - Consider the sequence $\left(\Phi_{n}, \Psi_{n}\right)$ given by

$$
\Phi_{n}(y, \tau)=\Phi(y, \tau+n), \quad \Psi_{n}(y, \tau)=\Psi(y, \tau+n) .
$$

Since $\Phi+\Psi$ is bounded, there exists a subsequence (still denoted by $\left(\Phi_{n}, \Psi_{n}\right)$ ) such that $\lim _{n \rightarrow \infty} \Phi_{n}(\cdot, \tau)=\Phi^{*}(\cdot, \tau)$ and $\lim _{n \rightarrow \infty} \Psi_{n}(\cdot, \tau)=$ $\Psi^{*}(\cdot, \tau)$ uniformly on compact sets $|y| \leq R$. Clearly, $\left(\Phi^{*}, \Psi^{*}\right)$ solves (3.2). By hypothesis, one of the cases (1.18) or (1.20) holds. Assume that $\left(\Phi^{*}, \Psi^{*}\right) \neq(\Gamma, \gamma)$. Then, for any $\varepsilon>0$ and $\tau_{0}>0$, we would have that

$$
\left\|\Phi\left(\cdot, \tau_{0}+n\right)\right\|+\left\|\Psi\left(\cdot, \tau_{0}+n\right)\right\| \leq \varepsilon, \quad \text { for large enough } n .
$$

Arguing as in the proof of Lemma 3.3, it then turns out that

$$
\|\Phi(\cdot, \tau)\| \leq C e^{-\frac{p+1}{p q-1} \tau},\|\Psi(\cdot, \tau)\| \leq C e^{-\frac{q+1}{p q-1} \tau}, \quad \text { as } \tau \rightarrow \infty .
$$

We then take up an argument already used in the proof of Lemma 3.5. We set

$$
G=|\Phi| e^{\frac{p+1}{p q-1} \tau}+|\Psi| e^{\frac{q+1}{p q-1} \tau},
$$

and readily check that $G$ satisfies

$$
G_{+} \leq \Delta G-\frac{1}{2} y \nabla G+C G
$$

for some $C>0$. We now consider the following integral introduced in [27]: For any $q>1$ and $r>0$, and for any $h \in L_{w}^{q}\left(\mathbf{R}^{N}\right)$ we write

$$
L_{r}^{q}(h)=\sup _{|\xi| \leq r}\left(\int_{\mathbf{R}^{N}}|h(y)|^{q} \exp \left(-\frac{(y-\xi)^{2}}{4}\right) \mathrm{d} \xi\right)^{1 / q} .
$$


Let $A_{0}, S_{0}(\tau)$ be as in the proof of Lemma 3.5. It has been proved in [27] (cf Section 2 therein) that

$$
L_{r}^{2}\left(S_{0}(\tau) \psi\right) \leq C^{*} L_{0}^{2}(\psi), \quad \text { for any } \tau>\delta \text { and some } C^{*}=C^{*}(\delta)>0
$$

From (4.2), (4.3) and (4.4) we then obtain that

$$
L_{1}^{2}(G(\cdot, \tau)) \leq C, \quad \text { for some } C>0, \text { and } \tau>0,
$$

which in turn implies

$$
L_{1}^{2}(\Phi(\cdot, \tau)) \leq C e^{-\frac{p+1}{p q-1} \tau}, \quad L_{1}^{2}(\Psi(\cdot, \tau)) \leq C e^{-\frac{q+1}{p q-1} \tau}
$$

for some positive generic constant $C$. We are thus led to

$$
\begin{aligned}
& \left\|\Phi_{\xi}(\cdot, \tau)\right\| \leq C e^{-\frac{p+1}{p q-1} \tau}, \quad\left\|\Psi_{\xi}(\cdot, \tau)\right\| \leq C e^{-\frac{q+1}{p q-1} \tau}, \\
& \text { for large } \tau, \text { where }|\xi| \leq 1 \\
& \text { and } \Phi_{\xi}(y, \tau)=\Phi(y+\xi, \tau), \Psi_{\xi}(y, \tau)=\Psi(y+\xi, \tau) .
\end{aligned}
$$

We can perform now a translation in space to see that, after selecting $\left(\Phi_{\xi}(\cdot, \tau), \Psi_{\xi}(\cdot, \tau)\right)$ as a new initial value in (3.2), standard regularity theory for parabolic equations will give

$$
0 \leq u(x, t)+v(x, t) \leq C, \text { for any }|x| \leq \delta<1 \text {, and any } \tau \in(T-\delta, T),
$$

which provides a contradiction with the assumptions of $(x, t) \equiv(0, T)$ being a blow up point. This concludes the proof.

As soon as Lemma 4.1 has been obtained, we are able to use the arguments developed in Section 3 to deal with the case where ( $3.4 a$ ) holds. Namely, we linearise as in (3.22) and make use of the functional frame previously implemented to repeat the steps in [26] where the scalar case (1.1) was analysed. This yields at once Theorem 3.

We next set out to prove that the profiles described in (1.29) actually occur.

\subsection{The proof of Theorem 4}

We shall obtain the sought-for result by means of a topological argument. Such approach has been employed by Alberto Bressan to discuss on blow up patterns in semilinear parabolic equations (cf. [7], [8]). Arguments alike to those to be explained below have been used in [23], [29]. We shall refer to 
these works for details, and concentrate instead in describing here the main idea behind the proof. Basically we will prove that if the coefficients $\alpha_{0}, \ldots$, $\alpha_{n}, \beta_{0}, \ldots, \beta_{\ell}$ defined below are chosen satisfying (4.11) and $v$ satisfies (4.46) then we recover (4.46) with a strict inequality for $\tau_{0} \leq \tau \leq \tau_{1}$, $\tau_{1} \geq \tau_{0}$ arbitrarily large. This allows us to use a topological argument that proves that (4.11) still holds for suitable $\alpha_{k}, \beta_{k}$ and $\tau_{1}$ replaced by $\tau_{1}+\delta$. By means of a continuation argument we could then assume that $\tau_{1}=+\infty$ and this yields the required result.

To simplify a bit the notation, we shall consider in detail the case $N=1$ only. It will become apparent from our approach, though, that the case of radial solutions in higher dimensions can be obtained at the expense of some minor modifications, which shall be omitted. To begin with, let $m$ be a fixed even integer, $m \geq 2$ and let $\ell$ be a positive integer such that

$-\left(\sigma+\frac{m}{2}\right)>1-\frac{\ell}{2}>-2\left(\sigma+\frac{m}{2}\right), \quad$ where $\sigma=(p+1)(q+1) /(p q-1)$.

Notice that such a $\ell$ always exists, since $\sigma>1$. Remark also that (4.7) can be restated in the form

$$
2+2 \sigma+m<\ell<2+4 \sigma+2 m \text {. }
$$

For fixed $\tau_{0}>1$, we now consider the following function

$$
\begin{aligned}
v_{0}\left(y, \tau_{0}\right)= & \alpha_{m} \bar{H}_{m}(y) e^{-\left(\sigma+\frac{m}{2}\right) \tau_{0}} u_{-} \\
& +\sum_{m \neq k \leq n} \alpha_{k} \bar{H}_{k}(y) e^{-\left(\sigma+\frac{k}{2}\right) \tau_{0}} u_{-} \\
& +\sum_{j=0}^{\ell-1} \beta_{j} \bar{H}_{j}(y) e^{\left(1-\frac{j}{2}\right) \tau_{0}} u_{+}+\beta_{\ell} \bar{H}_{\ell} e^{\left(1-\frac{\ell}{2}\right) \tau_{0}} u_{+}
\end{aligned}
$$

Here $\bar{H}_{j}(y)$ denotes a truncated version of the modified Hermite polynomial $H_{j}(y)$, i.e.,

$$
\bar{H}_{j}(y)= \begin{cases}H_{j}(y), & \text { for }|y| \leq C e^{\left(\frac{1}{2}-\frac{1}{\ell}\right) \tau_{0}} \\ K, & \text { for }|y| \geq 2 C e^{\left(\frac{1}{2}-\frac{1}{\ell}\right) \tau_{0}}\end{cases}
$$

where $C, K$ are large (but otherwise fixed) constants, $n$ is the largest integer which is equal or inferior to $2\left(\sigma+\frac{m}{2}\right)$, and $\alpha_{0}, \ldots, \alpha_{n}, \beta_{0}, \ldots, \beta_{\ell}$ are real constants to be selected presently. We certainly can rewrite (4.8) in the form

$$
\begin{aligned}
v_{0}\left(y, \tau_{0}\right) & \equiv v_{0}\left(y, \tau_{0}, \alpha_{0}, \ldots, \alpha_{n}, \beta_{0}, \ldots, \beta_{\ell}\right) \\
& =\alpha_{m} H_{m}(y) e^{-\left(\sigma+\frac{m}{2}\right) \tau_{0}} u_{-}+\sum_{m \neq k \leq n} \alpha_{k} H_{k}(y) e^{-\left(\sigma+\frac{k}{2}\right) \tau_{0}} u_{-}
\end{aligned}
$$




$$
\begin{aligned}
& +\sum_{j=0}^{\ell-1} \beta_{j} H_{j}(y) e^{\left(1-\frac{j}{2}\right) \tau_{0}} u_{+} \\
& +\beta_{\ell} H_{\ell} e^{\left(1-\frac{\ell}{2}\right) \tau_{0}} u_{+}+E\left(y, \tau_{0}\right),
\end{aligned}
$$

where $E\left(y, \tau_{0}\right) \equiv 0$ for $|y| \leq C e^{\left(\frac{1}{2}-\frac{1}{\ell}\right) \tau_{0}}$ and

$$
\left\|E\left(\cdot, \tau_{0}\right)\right\|=O\left(e^{-2\left(\sigma+\frac{m_{2}}{2}\right) \tau_{0}}\right) .
$$

Let us define now the function $v(y, \tau)$ as the solution of the Cauchy problem

$$
\begin{gathered}
v_{\tau}=A v+f(v), \quad \text { for } \tau>\tau_{0}, \\
v\left(\cdot, \tau_{0}\right)=v_{0}\left(y, \tau_{0}\right)
\end{gathered}
$$

(cf (3.23)). Fix now $\tau_{1}>\tau_{0}$, and let $\left(\alpha_{k}, \beta_{j}\right)$ with $k \neq m, j \neq \ell$ be selected so that

$$
\begin{array}{r}
\left\langle P_{-} v\left(\cdot, \tau_{1}\right), H_{k}\right\rangle=0, \quad \text { for } 0 \leq k \leq n, k \neq m, \\
\left\langle P_{+} v\left(\cdot, \tau_{1}\right), H_{j}\right\rangle=0, \quad \text { for } 0 \leq j \leq \ell-1 .
\end{array}
$$

We now claim that

For any $k$ such that $0 \leq k \leq n$ and $k \neq m$, there holds

$$
\left|\alpha_{k}\right|=O\left(e^{-\delta_{1} \tau_{0}}\right), \text { as } \tau_{0} \rightarrow \infty \text {, where } \delta_{1}=2\left(\sigma+\frac{m}{2}\right)-\left(\sigma+\frac{k}{2}\right)>0
$$

For any $j$ such that $0 \leq j \leq \ell-1$, there holds

$$
\left|\beta_{j}\right|=O\left(e^{-\delta_{2} \tau_{0}}\right), \text { as } \tau_{0} \rightarrow \infty, \text { where } \delta_{2}=2\left(\sigma+\frac{m}{2}\right)-\left(\frac{j}{2}-1\right)>0 \text {. }
$$

Let us show how to obtain $(4.12 a)$. To this end, we use variation of constants formula in $(4.10)$, to write $v(\cdot, \tau)$ in the form

$$
v(\cdot, \tau)=e^{A\left(\tau-\tau_{0}\right)} v\left(\cdot, \tau_{0}\right)+\int_{\tau_{0}}^{\tau} e^{A(\tau-s)} \int(v(\cdot, s)) \mathrm{d} s .
$$

We then set $\tau=\tau_{1}$ in (4.13), and observe that, for any fixed $k \neq m$ such that $0 \leq k \leq n$, condition (4.11a) yields

$$
\begin{aligned}
0 & =\alpha_{k} e^{-\left(\sigma+\frac{k}{2}\right) \tau_{1}}+\left\langle P_{-} \int_{\tau_{0}}^{\tau_{1}} e^{\Lambda\left(\tau_{1} s\right)} f(v(\cdot, s)) \mathrm{d} s, H_{k}\right\rangle+\left\langle P_{-} E\left(\cdot, \tau_{1}\right), H_{k}\right\rangle \\
& \equiv \alpha_{k} e^{-\left(\sigma+\frac{k}{2}\right) \tau_{1}}+R_{1}\left(\tau_{1}\right)+R_{2}\left(\tau_{1}\right)
\end{aligned}
$$


Using the fact that $f$ is quadratic, one readily sees that

$$
\left|R_{1}\left(\tau_{1}\right)\right| \leq C \int_{\tau_{0}}^{\tau_{1}} e^{-\left(\sigma+\frac{k}{2}\right)\left(\tau_{1}-s\right)} e^{-2\left(\sigma+\frac{m_{2}}{2}\right) s} \mathrm{~d} s \leq C e^{-\left(\sigma+\frac{k}{2}\right) \tau_{1}} e^{-\delta_{1} \tau_{0}},
$$

where $\left|R_{2}\left(\tau_{1}\right)\right|$ gives a contribution which is even smaller than the previous one. This yields at once (4.12a); the proof of $(4.12 b)$ is similar.

We next proceed to derive a priori bounds for $v(y, \tau)$, under the assumption that (4.11) holds. We do this by using variation of constants formula together with (4.9) to obtain that, for any $\tau \in\left[\tau_{0}, \tau_{1}\right]$,

$$
\begin{aligned}
v(y, \tau)= & \sum_{k \leq n} \alpha_{k} e^{-\left(\sigma+\frac{k}{2}\right) \tau} H_{k}(y) u_{-}+E_{1}(y, \tau) u_{-}+\sum_{j \leq \ell} \beta_{j} e^{\left(1-\frac{j}{2}\right) \tau} H_{j}(y) u_{+} \\
& +E_{2}(y, \tau) u_{+}+\int_{\tau_{0}}^{\tau} e^{A(\tau-s)} f(v(\cdot, s)) \mathrm{d} s \\
\equiv & S_{1}(y, \tau)+E_{1}(y, \tau) u_{-}+S_{2}(y, \tau) \\
& +E_{2}(y, \tau) u_{+}+S_{3}(y, \tau)
\end{aligned}
$$

where

$$
e^{A\left(\tau-\tau_{0}\right)} E(y, \tau)=E_{1}(y, \tau) u_{-}+E_{2}(y, \tau) u_{+} .
$$

We then write $S_{3}=S_{3}(y, \tau)$ as

$$
\begin{aligned}
S_{3}= & \sum_{k \leq n} H_{k} \int_{\tau_{0}}^{\tau} e^{-\left(\sigma+\frac{k}{2}\right)(\tau-s)}\left\langle P_{-} f, H_{k}\right\rangle \mathrm{d} s u_{-} \\
& +\sum_{k>n} H_{k} \int_{\tau_{0}}^{\tau} e^{-\left(\sigma+\frac{k}{2}\right)(\tau-s)}\left\langle P_{-} f, H_{k}\right\rangle \mathrm{d} s u_{-} \\
& +\sum_{j \leq \ell} H_{j} \int_{\tau_{0}}^{\tau} e^{\left(1-\frac{j}{2}\right)(\tau-s)}\left\langle P_{+} f, H_{j}\right\rangle \mathrm{d} s u_{+} \\
& +\sum_{j>\ell} H_{j} \int_{\tau_{0}}^{\tau} e^{\left(1-\frac{j}{2}\right)(\tau-s)}\left\langle P_{+} f, H_{j}\right\rangle \mathrm{d} s u_{+} .
\end{aligned}
$$

We now take advantage of (4.11a) and (4.15) to obtain that, for any $\tau \in\left[\tau_{0}, \tau_{1}\right]$,

$$
v(y, \tau)=-\sum_{m \neq k \leq n} H_{k} \int_{\tau_{0}}^{\tau_{1}} e^{A(\tau-s)}\left\langle P_{-} f, H_{k}\right\rangle \mathrm{d} s u_{-}
$$




$$
\begin{aligned}
& -\sum_{j \leq \ell-1} H_{j} \int_{\tau_{0}}^{\tau_{1}} e^{A(\tau-s)}\left\langle P_{+} f, H_{j}\right\rangle \mathrm{d} s u_{+} \\
& +\alpha_{m} e^{-\left(\sigma+\frac{m}{2}\right) \tau} H_{m}(y) u_{-}+\beta_{\ell} e^{\left(1-\frac{\ell}{2}\right) \tau} H_{\ell}(y) u_{+} \\
& +\sum_{k>n} H_{k} \int_{\tau_{0}}^{\tau} e^{-\left(\sigma+\frac{k}{2}\right)(\tau-s)}\left\langle P_{-} f, H_{k}\right\rangle \mathrm{d} s u_{-} \\
& +\sum_{j>\ell} H_{j} \int_{\tau_{0}}^{\tau} e^{\left(1-\frac{j}{2}\right)(\tau-s)}\left\langle P_{+} f, H_{j}\right\rangle \mathrm{d} s u_{+} \\
& +H_{m} \int_{\tau_{0}}^{\tau} e^{A(\tau-s)}\left\langle P_{-} f, H_{m}\right\rangle \mathrm{d} s u_{-} \\
& +H_{\ell} \int_{\tau_{0}}^{\tau} e^{A(\tau-s)}\left\langle P_{+} f, H_{\ell}\right\rangle \mathrm{d} s u_{+} \\
& +E_{1}(y, \tau) u_{-}+E_{2}(y, \tau) u_{+} .
\end{aligned}
$$

\section{Clearly}

$$
\begin{aligned}
& \left|\sum_{m \neq k \leq n} \int_{\tau}^{\tau_{1}} e^{A(\tau-s)}\left\langle P_{-} f, H_{k}\right\rangle \mathrm{d} s\right| \\
& \quad \leq C \sum_{m \neq k \leq n} \int_{\tau}^{\tau_{1}} e^{-\left(\sigma+\frac{k}{2}\right)(\tau-s)} e^{-2\left(\sigma+\frac{m_{2}}{2}\right) s} \mathrm{~d} s \leq C e^{-2\left(\sigma+\frac{m k}{2}\right) \tau},
\end{aligned}
$$

and moreover, using (4.7) and the fact that $f$ is quadratic,

$$
\begin{aligned}
& \left|\sum_{j<\ell} \int_{\tau}^{\tau_{1}} e^{A(\tau-s)}\left\langle P_{+} f, H_{j}\right\rangle \mathrm{d} s\right| \\
& \quad+\left|H_{m} \int_{\tau_{0}}^{\tau} e^{A(\tau-s)}\left\langle P_{-} f, H_{m}\right\rangle \mathrm{d} s\right| \\
& \quad+\left|H_{\ell} \int_{\tau_{0}}^{\tau} e^{A(\tau-s)}\left\langle P_{+} f, H_{\ell}\right\rangle \mathrm{d} s\right| \leq e^{-2\left(\sigma+\frac{m}{2}\right) \tau} .
\end{aligned}
$$

On the other hand, using Cauchy-Schwarz,

$$
\left\|\sum_{k>n} H_{k} \int_{\tau_{0}}^{\tau} e^{-\left(\sigma+\frac{h}{2}\right)(\tau-s)}\left\langle H_{-} f, H_{k}\right\rangle \mathrm{d} s\right\|
$$




$$
\begin{aligned}
& \leq C \sum_{k>n}\left(\int_{\tau_{0}}^{\tau} e^{-\left(\sigma+\frac{k}{2}\right)(\tau-s)}\left\langle P_{-} f, H_{k}\right\rangle \mathrm{d} s\right)^{2} \\
& \leq C\left(\sigma+\frac{n}{2}\right)^{-1} \int_{\tau_{0}}^{\tau} e^{-\left(\sigma+\frac{n}{2}\right)(\tau-s)}\left(\sum_{k>n}\left\langle P_{-} f, H_{k}\right\rangle^{2}\right) \mathrm{d} s \\
& \leq C\left(\sigma+\frac{n}{2}\right)^{-1} \int_{\tau_{0}}^{\tau} e^{-\left(\sigma+\frac{n}{2}\right)(\tau-s)} \mathrm{d} s \leq C\left(o+\frac{n}{2}\right)^{-1} e^{-2\left(\sigma+\frac{m}{2}\right) \tau} .
\end{aligned}
$$

As a matter of fact, the extra factor $(\sigma+n / 2)^{-1}$ on the right above yields at once

$$
\left\|\sum_{k>n} H_{k} \int_{\tau_{0}}^{\tau} e^{-\left(\sigma+\frac{k}{2}\right)(\tau-s)}\left\langle P_{-} f, H_{k}\right\rangle \mathrm{d} s\right\|_{H_{w}^{1}} \leq C e^{-2\left(\sigma+\frac{m}{2}\right) \tau} .
$$

Arguing in a similar way, we easily obtain

$$
\left\|\sum_{j>\ell} H_{j} \int_{\tau_{0}}^{\tau} e^{\left(1-\frac{j}{2}\right)(\tau-s)}\left\langle P_{+} f, H_{k}\right\rangle \mathrm{d} s\right\|_{H_{w}^{1}} \leq C e^{-2\left(\sigma+\frac{m}{2}\right) \tau} .
$$

Notice that in space dimension $N=1$ the above $H_{w}^{1}$-bounds give $L_{\mathrm{loc}}^{\infty}$ estimates hy Sobolev embeddings. Finally, one readily checks that the contribution corresponding to the terms $E_{1}(y, \tau), E_{2}(y, \tau)$ in (4.16) is of order $o\left(e^{-2\left(\sigma+\frac{m}{2}\right) \tau}\right)$ for $\tau \geq \tau_{0}$. We summarize our previous results (in particular, (4.12) and (4.17)) in the following

Lemma 4.2. - Assume that conditions (4.11) are satisfied. Then if $\tau_{0} \gg 1$, for any $\tau \in\left[\tau_{0}, \tau_{1}\right]$ one has that

$$
\begin{gathered}
v(y, \tau)=\alpha_{m} e^{-\left(\sigma+\frac{m}{2}\right) \tau} H_{m}(y) u_{-}+\beta_{\ell} e^{\left(1-\frac{\ell}{2}\right) \tau} H_{\ell}(y) u_{+}+O\left(e^{-2\left(\sigma+\frac{m}{2}\right) \tau}\right), \\
\text { uniformly on sets }|y| \leq C .
\end{gathered}
$$

Our next step consists in extending Lemma 4.2 (or rather a suitable version of it) to larger regions. To this end, we introduce a new variable $\xi$ given by

$$
\xi=y e^{\left(\frac{1}{2}-\frac{1}{2}\right) \tau} .
$$

In the variables $(\xi, \tau)$, the corresponding equations for $(\Phi, \Psi)$ read

$$
\frac{\xi}{\ell} \Phi_{\xi}=\Psi^{p}-\frac{p+1}{p q-1} \Phi+\left(e^{\left(\frac{2}{\ell}-1\right) \tau} \Phi_{\xi \xi}-\Phi_{\tau}\right),
$$




$$
\frac{\xi}{\ell} \Psi_{\xi}=\Phi^{q}-\frac{q+1}{p q-1} \Psi+\left(e^{\left(\frac{2}{\tau}-1\right) \tau} \Psi_{\xi \xi}-\Psi_{\tau}\right)
$$

Set now

$$
z=\ell \log |\xi|, \quad \text { so that } \dot{\Phi} \equiv \Phi_{z}=\frac{\xi}{\ell} \Phi_{\xi},
$$

and consider the autonomous system

$$
\begin{aligned}
& \dot{\Phi}=\Psi^{p}-\frac{p+1}{p q-1} \Phi \\
& \dot{\Psi}=\Phi^{q}-\frac{q+1}{p q-1} \Psi
\end{aligned}
$$

System (4.22) has two equilibria: $(0,0)$ and $(\Gamma, \gamma)$. A standard phase space analysis yields the following phase portrait for the corresponding trajectories

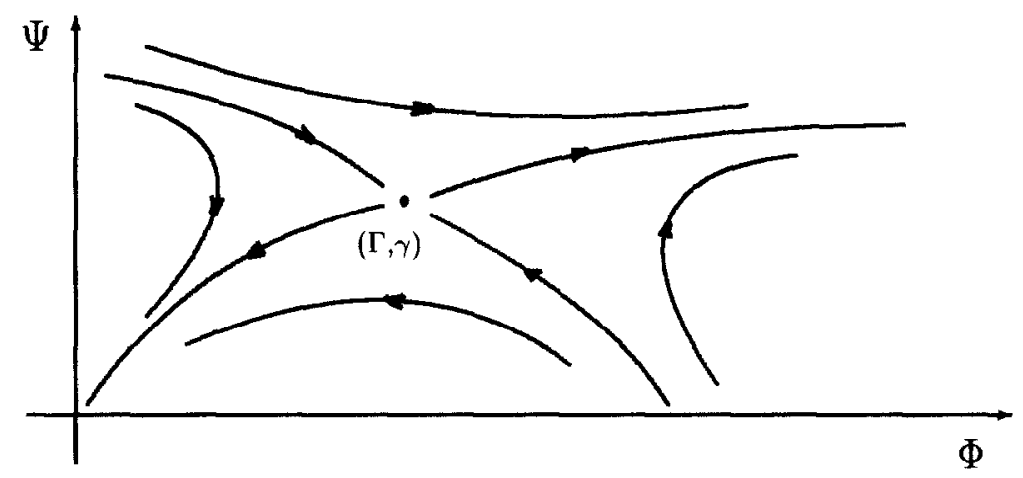

FIG 2. - Phase portrait of (4.22).

Let us denote by $\bar{v}=(\bar{\Phi}, \bar{\Psi})^{*}$ the stable trajectory linking the critical points $(\Gamma, \gamma)$ and $(0,0)$ in Figure 2. Classical linearisation procedures yield the asymptotic behaviours

$$
\begin{array}{ll}
\bar{\Phi}(\xi) \sim \Gamma-c_{1}|\xi|^{\ell}, & \text { as } \xi \rightarrow 0, \\
\bar{\Psi}(\xi) \sim \gamma-c_{2}|\xi|^{\ell}, & \text { as } \xi \rightarrow 0,
\end{array}
$$

for some positive constants $c_{1}, c_{2}$, and

$$
\bar{\Phi}(\xi) \sim K_{1}|\xi|^{-\ell \frac{p+1}{p q-1}}, \quad \text { as } \xi \rightarrow \infty
$$




$$
\bar{\Psi}(\xi) \sim K_{2}|\xi|^{-\ell \frac{q+1}{p q-1}}, \quad \text { as } \xi \rightarrow \infty,
$$

for some $K_{1}>0, K_{2}>0$. We now consider the following auxiliary function

$$
\begin{aligned}
v^{*}(\xi, \tau) & \equiv \bar{v}(\xi)+\alpha_{m} \bar{H}_{m}(\xi) e^{-\left(\sigma+\frac{m_{m}}{2}\right) \tau} u_{-}+R(\xi, \tau) \\
& \equiv \bar{v}(\xi)+W(\xi, \tau)+R(\xi, \tau)
\end{aligned}
$$

where

$$
R(\xi, \tau)=e^{\left(\frac{2}{\ell}-1\right) \tau} Q_{1}(\xi)+e^{-\left(\sigma+\frac{m}{2}\right) \tau} Q_{2}(\xi)+Q_{3}(\xi)
$$

and functions $Q_{i}(\xi)(i=1,2,3)$ are to be selected presently. As a a matter of fact, our choice of the $Q_{i}(\xi)$ 's will be made so that $v^{*}(\xi, \tau)$ will provide sub- and supersolutions of system (1.17) in regions $|\xi|=O(1)$. This goal will be achieved as follows. We set

$$
\mathcal{L}(u)=u^{\prime \prime}-\frac{1}{2} y u^{\prime}, \quad f=\left(f_{1}, f_{2}\right)^{*},
$$

where

$$
f_{1}(\Phi, \Psi)=\Psi^{p}-\frac{p+1}{p q-1} \Phi, \quad f_{2}(\Phi, \Psi)=\Phi^{q}-\frac{q+1}{p q-1} \Psi .
$$

We may thus rewrite (1.17) in the form

$$
v_{\tau}=\mathcal{L}(u)+f(v)
$$

If we rewrite now $v^{*}(\xi, \tau)$ in the variables $(y, \tau)$ and plug it into (4.27), we obtain that

$$
\bar{v}_{\tau}+W_{\tau}+R_{\tau}=\mathcal{L}(\bar{v}+W+R)+f(\bar{v}+W+R)+f(\bar{v})-f(\bar{v}) .
$$

Hence

$$
W_{\tau}+R_{\tau}=\mathcal{L}(W+R)+\bar{v}_{y y}+f(\bar{v}+W+R)-f(\bar{v})
$$

On the other hand, the arguments in Section 3 ( $c f$ for instance (3.22), (3.23) there) show that

$$
W_{\tau}=\mathcal{L}(W)+M(W)
$$

where $M$ is the matrix given in (3.25a). From (4.28) and (4.29) it follows Vol. 14, $\mathrm{n}^{\circ} 1-1997$ 


$$
R_{\tau}=\mathcal{L}(R)+\bar{v}_{y y}+f(\bar{v}+W+R)-f(\bar{v})-M(W)
$$

To obtain further insight, we split $f(v)$ into a linear and a nonlinear part. Namely, we set

$$
f(v)=\left(-\frac{p+1}{p q-1} \Phi, \frac{q+1}{p q-1} \Psi\right)^{*}+\left(\Psi^{p}, \Phi^{q}\right)^{*} \equiv \ell(v)+g(v) .
$$

From (4.30) and (4.31), we finally obtain

$$
\begin{aligned}
R_{\tau} & =\mathcal{L}(R)+\ell(R)+g(\bar{v}+W+R)-g(\bar{v})-(M-\ell) W+\bar{v}_{y y} \\
& \equiv A(R)+K(\xi, \tau)
\end{aligned}
$$

Write now

$$
\bar{M}(\xi)=\left(\begin{array}{cc}
\frac{p+1}{p q-1} & -p \bar{\Psi}(\xi)^{p-1} \\
-q \bar{\Phi}(\xi)^{q-1} & \frac{q+1}{p q-1}
\end{array}\right)
$$

We are now prepared to select $Q_{1}(\xi)$ and $Q_{2}(\xi)$ in (4.25). To start with, $Q_{1}(\xi)$ is a solution of

$$
-\left(1-\frac{2}{\ell}\right) Q_{1}+\frac{\xi}{\ell}\left(Q_{1}\right)_{\xi}+\bar{M}(\xi) Q_{1}=-\left(\bar{\Phi}_{\xi \xi}, \bar{\Psi}_{\xi \xi}\right)^{*} . \quad \text { for } \xi>0,(4.34 a)
$$

so that

$$
Q_{1}(\xi) \sim c \xi^{\ell-2} . \quad \text { as } \xi \rightarrow 0, \text { for some } c=\left(c_{1}, c_{2}\right)^{*}, c \neq 0
$$

On the other hand, $Q_{2}(\xi)$ is a solution of

$$
-\left(\sigma+\frac{m}{\ell}\right) Q_{2}+\frac{\xi}{\ell}\left(Q_{2}\right)_{\xi}+\vec{M}(\xi) Q_{2}=P(\xi) . \quad \text { for } \xi>0
$$

where

$$
P(\xi)=\left(\left(\bar{\Psi}^{p-1}-\gamma^{p-1}\right) W_{2}(\xi),\left(\bar{\Phi}^{q-1}-\Gamma^{q-1}\right) W_{1}(\xi)\right)^{*}
$$

with

$$
W=\left(W_{1}, W_{2}\right)^{*}
$$

as in $(4.25)$, so that

$$
Q_{2}(\xi) \sim d \xi^{m+\sigma \ell}, \quad \text { as } \xi \rightarrow 0, \text { for some } d=\left(d_{1}, d_{2}\right)^{*}, d \neq 0
$$


For $i=1,2$, let us write $Q_{i}(\xi)=\left(Q_{i}^{1}(\xi), Q_{i}^{2}(\xi)\right)^{*}$, and set $\alpha=\left(\alpha_{1}, \alpha_{2}\right)^{*}$. We shall use henceforth the following notation

$$
\alpha Q_{i}(\xi)=\left(\alpha_{1} Q_{i}^{1}(\xi), \alpha_{2} Q_{i}^{2}(\xi)\right)^{*}
$$

We shall prove now the following result

LEMMA 4.3. - There exist constants $\alpha=\left(\alpha_{1}, \alpha_{2}\right)^{*}$ and $\beta=\left(\beta_{1}, \beta_{2}\right)^{*}$ such that the function $R(\xi, \tau)$ given by

$$
R(\xi, \tau)=e^{\left(\frac{2}{\ell}-1\right) \tau} \alpha Q_{1}(\xi)+e^{-\left(\sigma+\frac{m}{2}\right) \tau} \beta Q_{2}(\xi)
$$

with $Q_{1}, Q_{2}$ as in (4.34), (4.35) respectively, satisfies

$$
R_{\tau}-\Lambda(R) \geq K(\xi, \tau)
$$

provided that $A e^{\left(\frac{1}{\ell}-\frac{1}{2}\right) \tau} \leq|\xi| \leq \delta$, where $A \gg 1,0<\delta \ll 1$ and $\tau \gg 1$, the inequality in (4.37) being understood as holding for each component of the vectors therein considered.

Proof. - A quick check reveals that, if $A e^{\left(\frac{1}{\ell}-\frac{1}{2}\right) \tau} \leq|\xi| \leq \delta$, where $A, \delta$ and $\tau$ are as before, then there holds $W+R \ll \bar{v}$. Setting $R=\left(R_{1}, R_{2}\right)^{*}$, (4.42) reads

$$
\begin{aligned}
\left(R_{1}\right)_{\tau}= & \frac{\xi}{\ell}\left(R_{1}\right)_{\xi}-\frac{p+1}{p q-1} R_{1}+p \bar{\Psi}^{p-1} R_{2}+p\left(\bar{\Psi}^{p-1}-\gamma^{p-1}\right) W_{2} \\
& +e^{\left(\frac{2}{\ell}-1\right) \tau}\left(\left(R_{1}\right)_{\xi \xi}+\bar{\Phi}_{\xi \xi}\right) \\
\left(R_{2}\right)_{\tau}= & \frac{\xi}{\ell}\left(R_{2}\right)_{\xi}-\frac{q+1}{p q-1} R_{2}+q \bar{\Phi}^{q-1} R_{1}+q\left(\bar{\Phi}^{q-1}-\Gamma^{q-1}\right) W_{1} \\
& +e^{\left(\frac{2}{\ell}-1\right) \tau}\left(\left(R_{2}\right)_{\xi \xi}+\bar{\Psi}_{\xi \xi}\right) .
\end{aligned}
$$

A routine computation reveals now that, if we set $R=e^{\left(\frac{2}{\ell}-1\right) \tau} \alpha Q_{1}$, we may select $\alpha$ so that

$$
R_{\tau}-\frac{\xi}{\ell} R_{\xi}-\bar{M}(\xi) R \gg e^{\left(\frac{2}{\ell}-1\right) \tau}\left(R_{\xi \xi}+\bar{\Phi}_{\xi \xi}\right)
$$

In deriving this last inequality, we make use of expansions (4.23) together with the fact that

$$
e^{2\left(\frac{2}{\ell}-1\right) \tau} \xi^{\ell-4} \ll e^{\left(\frac{2}{f}-1\right) \tau} \xi^{\ell-2}, \quad \text { whenever }|y| \geq A \gg 1
$$

Vol. 14, n ${ }^{\circ} 1-1997$ 
A similar argument shows then that if we set $R=e^{-\left(\sigma+\frac{m}{2}\right) \tau} \beta Q_{2}(\xi)$, we may select $\beta$ so that, in the region under consideration

$$
R_{\tau}-\frac{\xi}{\ell} R_{\xi}-\bar{M}(\xi) R \gg P(\xi)+e^{\left(\frac{2}{\ell}-1\right) \tau} R_{\xi \xi}
$$

Putting together (4.39) and (4.40), the Lemma follows.

Let now $B \gg 1$ be given, and let $Q_{\xi}$ be such that

$$
\begin{aligned}
& \frac{\xi}{\ell}\left(Q_{\xi}\right)_{\xi}+\bar{M}(0) Q_{\xi}=2\left(1-\chi_{B}\right)\left(\xi^{-\mu \ell}, \xi^{-\nu \ell}\right)^{*} . \quad \text { for } \xi \geq B \\
& \text { where } \mu=p(q+1) /(p q-1), \quad \nu=q(p+1) /(p q-1)
\end{aligned}
$$

and

$$
Q(B)=0, \quad Q_{\xi}(B) \neq 0 .
$$

In $(4.41 a)$ we are using the customary notation $\chi_{B}(\xi)$ to denote a function such that $\chi_{B}(\xi)=1$ for $\xi<B$ and $\chi_{B}(\xi)=0$ for $\xi>B$. We then have

Lemma 4.4. - Let $\tilde{R}(\xi, \tau)=R(\xi, \tau)+Q_{\xi}(\xi, \tau)$, where $R(\xi, \tau)$ is the function in the statement of Lemma 4.3. Then there holds

$$
\tilde{R}_{\tau}-\Lambda(\tilde{R}) \geq K(\xi, \tau), \quad \text { whenever } \delta \leq|\xi| \leq \delta e^{\frac{\tau}{\epsilon}}, \text { and } \tau \gg 1
$$

Proof. - It suffices to consider the case where $|\xi| \geq B$. To begin with, we observe that if $Q_{0}(\xi)$ solves the homogeneous version of (4.41a), one has that

$$
Q_{0}(\xi) \sim c\left(\xi^{-\ell \frac{p+1}{p q-1}} \cdot \xi^{\left.-\ell \frac{q+1}{p q-1}\right)^{*}} . \quad \text { as } \xi \rightarrow \infty,\right.
$$

for some $c=\left(c_{1}, c_{2}\right)^{*}(c f(4.23))$. An application of variation of constants formula in $(4.41 a)$ yields then that $Q_{\xi}(\xi)$ also has the asymptotic behaviour described in (4.43). We then observe that, as far as $|\xi| \leq \delta e^{\frac{\tau}{\xi}}$ with $\delta>0$ small enough, we have that $\bar{v}+W+R \leq 2 \bar{v}$, and (4.32) reads

$$
R_{\tau} \leq \mathcal{L}(R)+\ell(R)+g(3 \bar{v})+\text { (lower order terms) }
$$

whereupon $Q_{\xi}$ satisfies

$$
\left(Q_{\xi}\right)_{\tau}-\Lambda\left(Q_{\xi}\right) \geq g(3 \bar{v}), \quad \text { if } \delta \leq|\xi| \leq \delta e^{\bar{T}}, \tau \gg 1
$$

To conclude the proof of the Lemma, one has yet to prove that $R(\xi, \tau) \leq \delta_{1} Q_{\xi}(\xi, \tau)$, for some $\delta_{1}>0$ sufficiently small, in the region under consideration. To this end, we consider the first component of $R(\xi, \tau)$, 
which we shall still denote by $R$ for the ease of notation. Bearing this convention in mind, we notice that, as $\xi \rightarrow \infty$,

$$
e^{\left(\frac{2}{\ell}-1\right) \tau} Q_{1}(\xi) \sim e^{\left(\frac{2}{\ell}-1\right) \tau} \xi^{\ell-2} \xi^{-\ell \frac{p+1}{p q-1}} \leq \delta^{\ell-2} \xi^{-\ell \frac{p+1}{p q-1}}
$$

In a similar way, we see that $e^{-\left(\sigma+\frac{m}{2}\right) \tau} Q_{2}(\xi) \leq \delta^{m+\sigma \ell} \xi^{-\ell \frac{p+1}{p q-1}}$, as $\xi \rightarrow \infty$. The second component of $R(\xi, \tau)$ is taken care of in the same way, and the result follows.

For convenience, we next point out a consequence of our previous results. We remark first that the region where (4.42) holds corresponds to $|y| \leq \delta e^{\frac{\tau}{2}}$, $\tau \gg 1$ (i.e., $|x| \leq \delta$ and $\tau \sim T$ ). Consider now the subregion given by

$$
\Sigma_{1}=\left\{\frac{\delta}{2} e^{\frac{\tau}{2}} \leq|y| \leq \delta e^{\frac{\tau}{2}}, \tau \geq \tau_{0} \gg 1\right\}
$$

One easily checks that $R+Q \ll \bar{v}$ in $\Sigma_{1}$. Recalling (4.25), it turns out that CoRollary 4.5. - In $\Sigma_{1}$ there holds

$$
R(y, \tau)+Q_{3}(y, \tau)+\bar{v}(y, \tau) \sim\left(\begin{array}{l}
K_{1} y^{-\ell \frac{p+1}{p q-1}} e^{\left(\frac{\ell}{2}-1\right) \frac{p+1}{p q-1} \tau} \\
K_{2} y^{-\ell \frac{q+1}{p q-1}} e^{\left(\frac{\varepsilon}{2}-1\right) \frac{q+1}{p q-1} \tau}
\end{array}\right)
$$

for some positive constants $K_{1}$ and $K_{2}$.

End of the proof of Theorem 4. - Let $\vartheta \leq 1$ be a given positive number, and let us define $\mathcal{A}^{\vartheta} \equiv \mathcal{A}^{\vartheta}\left(\tau_{0}, \mathcal{C}, \mathcal{K} ; m, \ell\right)$ as the set of smooth functions $h(y, \tau)=\left(h_{1}, h_{2}\right)^{*}$ such that the following properties hold

$$
\begin{aligned}
& |h(y, \tau)| \leq \vartheta C\left(1+|y|^{m}\right) e^{-\left(\sigma+\frac{m}{2}\right) \tau}, \quad \text { when } \quad 0 \leq|y| \leq C, \quad \tau \geq \tau_{0}, \\
& \left|h_{1}(y, \tau)\right| \leq \vartheta C|y|^{-\ell \frac{p+1}{p q-1}} e^{\left(\frac{\xi}{2}-1\right) \frac{p+1}{p q-1} \tau}, \quad \text { when } C \leq|y| \leq \delta e^{\frac{\tau}{2}}, \quad \tau \geq \tau_{0} \text {. } \\
& \left|h_{2}(y, \tau)\right| \leq \vartheta C|y|^{-\ell \frac{q+1}{p q-1}} e^{\left(\frac{\ell}{2}-1\right) \frac{q+1}{p q-1} \tau}, \quad \text { when } \quad C \leq|y| \leq \delta e^{\frac{\tau}{2}}, \quad \tau \geq \tau_{0}, \\
& |h(y, \tau)| \leq \vartheta K, \quad \text { when }|y| \geq \delta e^{\frac{\tau}{2}}, \quad \tau \geq \tau_{0}
\end{aligned}
$$

We now define

$\mathcal{A}_{\tau_{0}, \tau_{1}}^{\vartheta}$ is the set of functions satisfying (4.46) whenever $\tau \in\left[\tau_{0}, \tau_{1}\right]$,

and argue as follows 
Step 1. - Take $\tau_{0} \gg 1$ large enough, and select functions $\Phi\left(y, \tau_{0}\right)$, $\Psi\left(y, \tau_{0}\right)$ not identically zero and such that

$$
\begin{aligned}
& \Phi\left(y, \tau_{0}\right)<\frac{K_{1}}{2}|y|^{-\ell \frac{p+1}{p q-1}} e^{\left(\frac{\ell}{2}-1\right) \frac{p+1}{p q-1} \tau_{0}}, \quad \text { for } \frac{\delta}{2} e^{\frac{\tau_{0}}{2}} \leq|y| \leq \delta e^{\frac{\tau_{0}}{2}} . \\
& \Psi\left(y, \tau_{0}\right)<\frac{K_{2}}{2}|y|^{-\ell \frac{q+1}{p q-1}} e^{\left(\frac{\ell}{2}-1\right) \frac{q+1}{p q-1} \tau_{0}}, \quad \text { for } \frac{\delta}{2} e^{\frac{\tau_{0}}{2}} \leq|y| \leq \delta e^{\frac{\tau_{0}}{2}} .
\end{aligned}
$$

Since $(\Phi, \Psi)$ are sufficiently small in the interval

$$
I_{0}=\left\{y\left|\frac{\delta}{2} e^{\frac{\tau_{0}}{2}} \leq\right| y \mid \leq \delta e^{\frac{\tau_{0}}{2}}\right\}
$$

a typical degeneracy argument repeatedly used in this work (cf. for instance that in the proof of Lemma 4.1) yields then that there is no blow up in, say, the subset

$$
I_{0}^{\prime}=\left\{y\left|\frac{2}{3} \delta e^{\frac{\tau_{0}}{2}} \leq\right| y \mid \leq \frac{3}{4} \delta e^{\frac{\tau_{0}}{2}}\right\}
$$

Step 2. - Let $\tau_{1}>\tau_{0}$, and select $v_{0}\left(y, \tau_{0}\right)$ given by (4.8), where constants $\left(\alpha_{k}, \beta_{j}\right)$ are selected so that (4.11) holds, and such that (4.48) and (4.49) are satisfied. The corresponding solution $v(y, \tau)$ of (4.10) satisfies $v(y, \tau) \in \mathcal{A}_{\tau_{0}, \tau_{1}}^{\vartheta}$ for some $\vartheta<1$. In particular, $v(y, \tau)$ remains bounded for $0 \leq|y| \leq \frac{3}{4} e^{\frac{\tau_{1}}{2}}, \tau_{0} \leq \tau \leq \tau_{1}$. We have shown in detail that (4.46a)-(4.46c) hold; the remaining bound $(4.46 d)$ is easily seen to hold. We now claim that $v(y, \tau) \in \mathcal{A}_{\tau_{0}, \tau}^{\vartheta}$ for all $\tau>\tau_{0}$. In view of our previous arguments, that will in turn ensure that $v(y, \tau)$ develops the blow up behaviour encoded in (1.29) as $\tau \rightarrow \infty$. To justify our claim, we just remark that, since $\vartheta<1$, standard continuous dependence results yield that there exists $\tau>\tau_{1}$ so that $v(y, \cdot) \in \mathcal{A}_{\tau_{0}, \tau}^{\frac{1+\vartheta}{2}}$. Let $\tau^{*}$ be the supremum of such $\tau$, and suppose that $\tau^{*}<\infty$. Then arguing as before we would obtain that $v(y, \cdot) \in \mathcal{A}_{\tau_{0}, \tau}^{\prime}$ with $\chi=\vartheta(1+\vartheta) / 2$, and therefore $v(y, \cdot) \in \mathcal{A}_{\tau_{0}, \tau}^{\frac{1+*}{2}}$ for some $\tau>\tau^{*}$, which provides a contradiction.

\section{REFERENCES}

[1] D. AMADORI, Unstable blow up patterns, to appear in Diff. and Integr. Equations.

[2] D. Andreucci, New results on the Cauchy problem for parabolic systems and equations with strongly nonlinear sources, Manuscripta Math., Vol. 77, 1992, pp. 127-159.

[3] D. ANDREuccl, Degenerate parabolic equations with initial data measures, to appear in Trans. Amer, Mat. Soc.

[4] D. Andreucci and E. DiBenedetto, On the Cauchy problem and initial traces for a class of evolution equations with strongly nonlinear sources, Ann. Sc. Normale Pisa, Vol. 18, 1991.

[5] J. Bebernes and A. LACEY, Finite-time blow up for a particular parabolic systern, SIAM J. Math. Anal., Vol. 21, no. 6, 1990, pp. 1415-1425. 
[6] J. BeBernes and A. LACEY, Finite-time blow up for semilinear reactive-diffusive systems, to appear in J. Diff. Eq.

[7] A. BRESSAN, On the asymptotic shape of blow up, Indiana Univ. Math. J., Vol. 39, no. 4 1990, pp. 947-960

[8] A. BresSAN, Stable blow up patterns, J. Diff. Equations, Vol. 98, 1992, pp. 57-75.

[9] J. BRICMONT and A. KuPIAINEN, Universality in blow up for nonlinear heat equations, preprint, 1993.

[10] G. CARISTI and E. Mitidieri, Blow up estimates of positive solutions of a parabolic system to appear in J. Diff. Eq.

[11] E. DiBenedetto, Degenerate parabolic equations, Springer-Verlag, New York, 1993.

[12] M. EsCOBEDo and M. A. HeRRERo, Boundedness and blow up for a semilinear reaction diffusion system, J. Diff. Eq., Vol. 89, no. 1, 1991, pp. 176-202.

[13] M. Escobedo and M. A. Herrero, A semilinear parabolic system in a bounded domain Annali Mat. Pura Appl. (IV), CLXV, 1993, pp. 315-336.

[14] M. EsCOBEDO and H. A. Levine, Critical blow up and global existence numbers for a weakly coupled system of reaction-diffusion equations, to appear in Trans. Amer. Math. Soc.

[15] S. FiLIPPAS and R. V. KoHN, Refined asymptotics for the blow up of $u_{t}-\Delta u=u^{p}$, Comm. Pure Appl. Math., Vol. 45, 1992, pp. 821-869.

[16] S. FilipPAS and F. MerLE, Modulation theory for the blow up of vector valued nonlinear heat equations, to appear in $J$. Diff. Eq.

[17] V. A. Galaktionov and S. A. Posashrov, Application of new comparison theorems in the investigation of unbounded solutions of nonlinear parabolic equations, Diff. Urav., Vol. 22, no. 7, 1986, pp. 1165-1173.

[18] B. Gidas and J. Spruck, Global and local behaviour of positive solutions of nonlinear elliptic equations, Comm. Pure Appl. Math., Vol. 34, 1981, pp. 525-598.

[19] Y. GiGA and R. V. KOHN, Asymptotically self-similar blow up of semilinear heat equations Comm. Pure Appl. Math., Vol. 38, 1985, pp. 297-319.

[20] Y. Giga, R.V. KoHN, Nondegeneracy of blow up for semilinear heat equations, Comm. Pure Appl. Math., Vol. 42, 1989, pp. 845-884.

[21] M. A. Herrern and J. J. I.. VeLÁZZQUUEZ, Blow up behaviour of one dimensional semilinear parabolic equations, Ann. Inst. H. Poincaré, Vol. 10, no. 2, 1993, pp. 131-189.

[22] M. A. Herrero and J. J. L. Velázquez, Flat blow up in one dimensional semilinear heat equations, Diff. and Integral Eq., Vol. 5, 1992, pp. 973-998.

[23] M. A. Herrero and J. J. L. VelázQuez, Explosion de solutions d'equations paraboliques semilinéaires supercritiques, C. R. Acad. Sci. Série A, Vol. 319, 1994, pp. 141-145.

[24] O. A. Ladyzenskaja, V. A. Solonnikov and N. N. Uraltseva, Linear and quasilinear equations of parabolic type, AMS Translations of Math., Monographs, XXIII, Providence RI, 1968.

[25] F. ROTHE, Global solutions of reaction-diffusion systems, in Lecture Notes in Mathematics, 1072, Springer-Verlag, New York, 1984

[26] J. J. L. VELÁZQUEZ, Classification of singularities for blowing up solutions in higher dimensions, Trans. Amer, Math. Soc., Vol. 338, no. 1, 1993, pp. 441-464.

[27] J. J. L. VELÁZQUeZ, Higher dimensional blow up for semilinear parabolic equations, Comm. in PDE, Vol. 17, no. 9\&10, 1992, pp. 1567-1596.

[28] J. J. L. VELÁzQUEZ, Blow up of semilinear parabolic equations, in Recent advances in partial differential equations, eds. M. A. Herrero and E. Zuazua, Research in Applied Mathematics, Masson \& J. Wiley, 1994, pp. 131-145.

[29] J. J. L. Velázquez, Curvature blow up in perturbations of minimizing cones evolving by mean curvature flow, Ann. Scuola Normale Sup. Pisa, Serie IV, Vol. XXI, 1994. pp. 595-628.

[30] F.B. Weisslek, An $L^{\infty}$ blow up estimate for a nolinear heal equation, Comm. Pure Appl. Math., Vol. 38, 1985, pp. 291-295.

(Manuscript received January 30, 1995.)

Vol. $14, n^{\circ} 1-1997$ 